\title{
Multi-disease Predictive Analytics: A Clinical Knowledge-aware Approach
}

LIN QIU, Department of Information Systems and Analytics, National University of Singapore

SRUTHI GORANTLA, Department of Computer Science, National University of Singapore

VAIBHAV RAJAN and BERNARD C. Y. TAN, Department of Information Systems and Analytics, National University of Singapore

Multi-Disease Predictive Analytics (MDPA) models simultaneously predict the risks of multiple diseases in patients and are valuable in early diagnoses. Patients tend to have multiple diseases simultaneously or develop multiple complications over time, and MDPA models can learn and effectively utilize such correlations between diseases. Data from large-scale Electronic Health Records (EHR) can be used through Multi-Label Learning (MLL) methods to develop MDPA models. However, data-driven approaches for MDPA face the challenge of data imbalance, because rare diseases tend to have much less data than common diseases. Insufficient data for rare diseases makes it difficult to leverage correlations with other diseases. These correlations are studied and recorded in biomedical literature but are rarely utilized in predictive analytics. This article presents a novel method called Knowledge-Aware Approach (KAA) that learns clinical correlations from the rapidly growing body of clinical knowledge. KAA can be combined with any data-driven MLL model for MDPA to refine the predictions of the model. Our extensive experiments, on real EHR data, show that the use of KAA improves the predictive performance of commonly used MDPA models, particularly for rare diseases. KAA is also found to be superior to existing general approaches of combining clinical knowledge with data-driven models. Further, a counterfactual analysis shows the efficacy of KAA in improving physicians' ability to prescribe preventive treatments.

\section{CCS Concepts: • Applied computing $\rightarrow$ Health informatics;}

Additional Key Words and Phrases: Electronic health records, diagnosis prediction, rare diseases, multi-label learning, knowledge graph, biomedical literature

\section{ACM Reference format:}

Lin Qiu, Sruthi Gorantla, Vaibhav Rajan, and Bernard C. Y. Tan. 2021. Multi-disease Predictive Analytics: A Clinical Knowledge-aware Approach. ACM Trans. Manage. Inf. Syst. 12, 3, Article 19 (May 2021), 34 pages. https://doi.org/10.1145/3447942

This article was supported by Singapore Ministry of Education Academic Research Fund Tier 1 [R-253-000-138-133]. Authors' addresses: L. Qiu (corresponding author), Department of Information Systems and Analytics, National University of Singapore, Computing Drive 15, 117418, Singapore; email: lin_qiu@u.nus.edu; S. Gorantla, Department of Computer Science and Automation, Indian Institute of Science, Mirinji Marg, Mathikere, Bengaluru, Karnataka 560012, Bangalore, India; email: gorantlas@iisc.ac.in; V. Rajan, Department of Information Systems and Analytics, National University of Singapore, Computing Drive 15, 117418, Singapore; email: vaibhav.rajan@nus.edu.sg; B. C. Y. Tan, Department of Information Systems and Analytics, National University of Singapore, Computing Drive 15, 117418, Singapore; email: btan@comp.nus.edu.sg. Permission to make digital or hard copies of all or part of this work for personal or classroom use is granted without fee provided that copies are not made or distributed for profit or commercial advantage and that copies bear this notice and the full citation on the first page. Copyrights for components of this work owned by others than ACM must be honored. Abstracting with credit is permitted. To copy otherwise, or republish, to post on servers or to redistribute to lists, requires prior specific permission and/or a fee. Request permissions from permissions@acm.org.

(c) 2021 Association for Computing Machinery.

2158-656X/2021/05-ART19 \$15.00

https://doi.org/10.1145/3447942 


\section{INTRODUCTION}

The ability to identify high-risk individuals prior to the onset of diseases facilitates personalized preventive care and reduces overall rates of morbidity and mortality. Compared with predicting diseases individually, it is more useful and realistic to predict multiple diseases, because patients tend to have multiple diseases simultaneously and patients with a single disease may develop other complications over time. For example, patients with diabetes are likely to have stroke, heart disease, and renal failure [8]. Rare diseases, which affect fewer than 200,000 patients in the U.S., are especially challenging to diagnose and lead to substantial clinical and economic burden [47].

Traditionally, physicians play the role of identifying potential diseases that patients may have, based on patients' health information and physicians' experience and knowledge. However, this process is challenging, because all physicians may not be equally knowledgeable or experienced to identify all possible diseases [2]. Physicians are usually trained in a specialized narrow domain and so, many potential diseases may be out of their scope of expertise, particularly rare diseases. Also, biomedical knowledge is being produced at a rapid rate-more than $0.8 \mathrm{M}$ new articles are added annually into the biomedical database MEDLINE [38]-and physicians, busy in their practice, may not always have up-to-date knowledge. Hence, reliable automated decision support tools that can aid physicians in accurate diagnoses is crucial. Diagnostic decision support systems, which use predictive models for diagnoses, have helped physicians play this role since the 1970s [36].

The availability of large-scale Electronic Health Records (EHR) has spurred the development of Machine Learning methods for a variety of clinical predictive tasks, including diagnosis prediction [43, 51]. Multi-label and multi-task learning models have been developed for multi-disease predictive analytics (MDPA), i.e., the simultaneous prediction of risks of multiple diseases. Such models can learn correlations across multiple diseases, from historical EHR data, to improve overall prediction. However, there are considerably more patient samples for common diseases (e.g., diabetes) in EHR, for a predictive model to learn from, than for rare diseases (e.g., Hodgkin's disease). Due to lack of sufficient samples, correlations between rare diseases and other diseases can be very difficult to capture.

A common approach to address the problem of insufficient samples is to use auxiliary information from Biomedical Knowledge Graphs (KG) to develop combined data-driven knowledgebased models. Knowledge graphs are large, heterogeneous information networks with multiple node types representing clinical concepts (e.g., diseases, drugs) and multiple edge types (e.g., "treats," "predisposes") representing associations between pairs of clinical concepts. These KGs are being actively developed by a combination of manual curation and automatic text mining from biomedical literature. Such combined data-driven knowledge-based models have been found to improve the accuracy and interpretability of diagnosis prediction [12, 34, 52]. However, previous studies have two limitations. Most of the models have been designed to use only a relatively small part of the knowledge graph containing hierarchical information, e.g., ICD disease classification hierarchy [44]. KGs contain considerably more information on, for example, adverse drug events, risk factors, and co-morbidities. Recognizing the need for modeling additional information, some recent works, such as Reference [52], have utilized complete KGs for (single) diagnosis prediction. But, they have specialized architectures that cannot be generalized easily for MDPA. Further, to our knowledge, no previous study has evaluated the efficacy of combined data-driven knowledge-based models for rare disease prediction.

In this article, we address these gaps through two contributions. First, we develop a general approach, called Knowledge Aware Approach (KAA), of using information from knowledge graphs in Multi-Label Learning (MLL) models. Unlike previous approaches where KGs are used at training time, our approach uses them to refine the predictions from a trained MLL model. By 


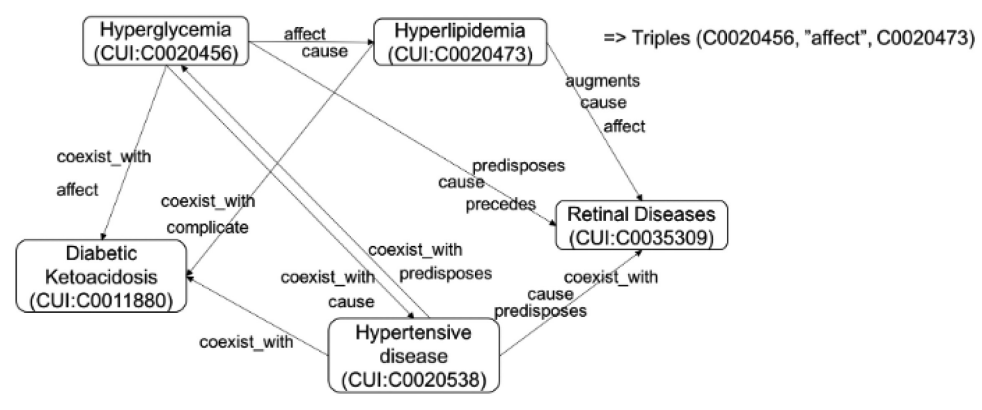

Fig. 1. Portion of Knowledge Graph constructed from SemMedDB triples.

thus decoupling the use of KGs from model training, KAA offers a unique and highly flexible approach that can be used with any underlying data-driven MDPA model. Second, we extensively evaluate the performance of several MLL models, with the use of KAA, for predicting multiple common and rare diseases on a large real EHR dataset. In addition to experiments to compare predictive accuracy, we also conduct a counterfactual analysis to evaluate the utility of predictive models to augment the physician's decision-making ability. Our experiments demonstrate the efficacy of KAA in improving the predictive accuracy of all the MLL models evaluated, particularly for rare diseases. Our KAA model can be found in https://bitbucket.org/q_lin/kaa.git/src.

\section{RESEARCH BACKGROUND}

\subsection{Literature-derived Knowledge Graphs}

Biomedical literature is the primary source of clinical knowledge, obtained from research (e.g., clinical trials) as well as experience (e.g., case studies). This literature can be freely accessed from databases such as PubMed, which contains more than 28M articles from MEDLINE [38]. There are ongoing efforts to create standardized vocabularies and ontologies to encode this vast body of biomedical literature. For instance, Metathesaurus from the Unified Medical Language System created by the U.S. National Library of Medicine is the largest thesaurus in the biomedical domain [1] that comprises over $1 \mathrm{M}$ biomedical concepts and $5 \mathrm{M}$ concept names. The Metathesaurus is organized by concepts represented by Controlled Unique Identifier (CUI) for biomedical vocabulary as well as relationships between the concepts such as "causes" or "co-exists with."

Manual creation of ontologies for the enormous amount of biomedical literature is infeasible. Thus, automated and scalable Natural Language Processing systems have been designed to encode knowledge from the primary literature directly [20]. SemMedDB is one such system that extracts "semantic predications" automatically from the titles and abstracts of all PubMed articles [28]. Each semantic prediction consists of a triple (subject, predicate, object). The subject and object are clinical concepts from the Metathesaurus. The predicate indicates a relationship between the subject and the object, such as "treats" or "causes." These triples can be viewed as directed edges and nodes in a knowledge graph, where each node corresponds to a subject or an object, labelled by their CUI, and each directed edge corresponds to a predicate, labelled by the relationship. Figure 1 shows part of a KG from SemMedDB.

SemMedDB includes 30 types of relationships (listed in Appendix A). The corresponding KG is dense, with about $94 \mathrm{M}$ predications (edges) [28]. Two clinical concepts may be connected by multiple paths (sequence of one or more edges) representing various direct and indirect relationships. To enable reasoning on such large, complex graphs and to automate feature engineering, knowledge graph embeddings have been developed that yield state-of-the-art results in many graph mining applications. These embeddings encode the global structural properties of the KG into vectorial 
representations of its vertices [26, 48]. In our experiments, we use one such method, TransE [4], to obtain vectorial representations of clinical concepts from SemMedDB. We provide more details on TransE in Appendix B. With such representations, similarity between diseases can be computed algebraically using vectorial measures of similarity, e.g., cosine similarity. A high similarity between diseases indicates that they have similar properties in the graph, e.g., similar neighborhood, that in turn would indicate shared clinical relations such as causes, treatments, or risk factors.

\subsection{Multi-disease Predictive Analytics}

Wide adoption of EHR in recent years has led to the development of many machine learning models for diagnosis prediction using historical EHR data comprising clinical investigations, drug prescriptions, previous diagnoses, and demographic information, e.g., References [11, 24, 31, 33]. Majority of the previous works on diagnosis prediction has focused on the prediction of specific single diseases, e.g., References [3, 13, 14, 55]. Data-driven models for MDPA can be formulated through multi-task learning (MTL) or multi-label learning (MLL). MTL views each patient as an example with multiple tasks (one disease per task) and trains the multiple tasks jointly with shared computational structure to improve learning, e.g., Reference [30]. MLL associates each patient with multiple labels (one label per disease). MLL is applicable for large label spaces while MTL is not, which makes MLL more suitable for health risk modelling, since the number of possible diseases can be large. Furthermore, MTL assumes that every example (patient) is associated with all tasks (diseases), while MLL allows each example (patient) to be associated with a subset of labels (diseases) [54], making MLL a more general model. Hence, most previous works on MDPA have used MLL models [39, 42, 49, 57].

There are numerous ways of building MLL models; a detailed review can be found in Reference [54]. We highlight two categories of methods that are most common in diagnosis prediction and later show how our proposed KAA method improves predictive accuracy in both cases. In the first category a model is trained for each disease, e.g., Binary Relevance [5]. This is equivalent to modeling each disease independently and we denote it by MDS (model diseases separately). In the second category of MLL methods, which we call MDJ (model diseases jointly), the training process is shared, i.e., the likelihood of each disease is learned jointly. Since all the disease labels are used together in the training process, correlations between the diseases are learned well. In both cases, all patient features are utilized during training and the final predictions are given for all diseases simultaneously. See Figure 2.

Using Biomedical Knowledge in Predictive Models. Clinical knowledge is essential to select important features for use in predictive models. To obviate the need for such manual feature engineering, which is cumbersome and time-consuming, most recent approaches have developed "end-to-end" models with minimal or no feature selection. Biomedical knowledge has been utilized in these models during training to improve the predictive accuracy and address the problem of insufficient samples for less common diseases.

The work of Reference [10] was an early deep neural network-based framework that can utilize knowledge-based priors to regularize parameters in their networks. They evaluated their model using only the ICD hierarchy and did not test its predictive accuracy for MDPA. Subsequent work developed more specialized neural architectures to effectively utilize the hierarchical structure of disease ontologies to obtain clinical concept embeddings. For instance, GRAM uses an attentionbased mechanism that gives more weight to an ancestor of a clinical concept that has less frequency in the data. This allows it to learn from related diseases guided by the parent-child relations in the disease hierarchy [12]. In GRAM, the ICD hierarchy is only used while learning disease representations, while KAME [34] uses it additionally in generating hospital visit embeddings. Both these 


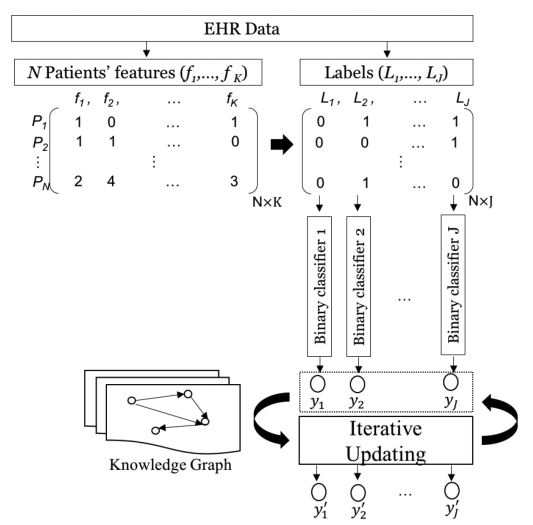

(a) For MDS

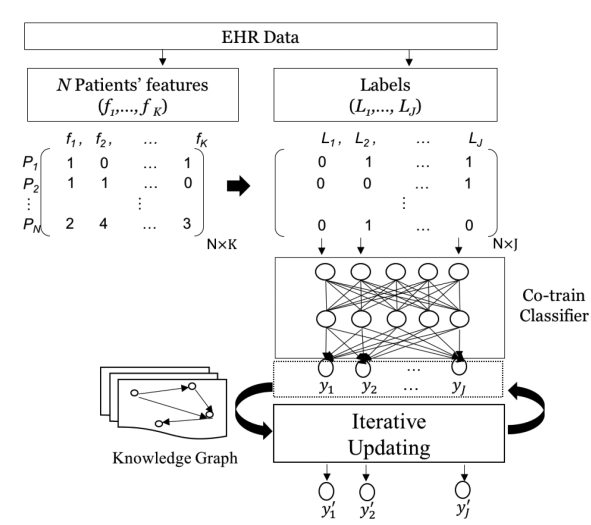

(b) For MDJ

Fig. 2. Our KAA applied to MLL predictions.

works evaluate next-visit diagnoses prediction, but they do not specifically evaluate accuracy of rare disease prediction. Further, both these models can use only hierarchical information from disease ontologies and not the entire KG. Recently, DG-RNN was proposed by Reference [52], which uses KG embeddings to obtain attention weights for each clinical concept used for prediction. They initialize the embeddings with TransE embeddings from the entire KG and then fine-tune the embeddings using neighbors (from the KG) of each clinical concept found in the data. For this crucial step of neighborhood selection, only disease nodes and two kinds of edges that are most relevant to heart failure prediction are used from the KG. Without such task-specific selection, each clinical event will have a very large number of potentially less useful neighbors in the dense KG, leading to difficulties in training and loss of interpretability. Further, their DG-RNN has been designed for risk prediction of a single disease (heart failure) and not evaluated for MLL, which may require additional model development, as discussed in Reference [31].

Among these approaches the most general approach, with respect to use of KGs, is that of Reference [10]. Their model can use auxiliary information of label similarity from any source, including knowledge graph embeddings. We denote this method by MDJ-Regu and provide more details in Appendix C. All the previous approaches are closely tied to the underlying data-driven model architectures. MDJ-Regu can only be used with neural network based MDPA models. GRAM, KAME and DG-RNN have even more specialized neural architectures, as described above.

Another general approach of using auxiliary knowledge in predictive models is to use pretrained embeddings as features. This has been used in many applications, not just in MDPA. For example, knowledge graph embeddings of drugs and diseases have been used to predict adverse drug events [35]. Such an approach can be used with both MDS and MDJ, and we call them MDS-Embed and MDJ-Embed, respectively, where the embeddings are obtained using TransE.

In contrast to all these models, where KGs are used during model training, our proposed KAA is a general framework that uses KGs to refine the predictions from an MLL model. Hence, it can be applied on the predictions obtained from any MLL model.

\section{MODEL DEVELOPMENT}

We now describe our KAA, which takes disease prediction probabilities from any MLL method as input and refines them using a knowledge graph to produce new prediction probabilities. 


\subsection{Notation and Problem Setting}

Let $N$ be the number of patients in the training data, $K$ be the dimensionality of the feature set, and $J$ be the total number of labels. Each patient is associated with a $K$-dimensional feature vector that encodes patients' features, such as medications and laboratory investigation results, extracted from historical EHR data. Each patient is diagnosed with a set of diseases and the $J$-dimensional binary label vector encodes this information: 1 indicates presence of a disease and 0 indicates absence. We denote the label for the $j$ th disease by $y_{j}(1 \leq j \leq J)$.

After an MLL method is trained on historical data of $N$ patients, it can predict the probabilities of the $J$ labels given any $K$-dimensional feature vector $x$ of a patient. In the MDS category, independent classifiers $g_{j}(1 \leq j \leq J)$ are learned separately, e.g., through Binary Relevance. After training, $g_{j}$ can produce the probability $P_{j}$ of the $j$ th disease (i.e., the probability of label $y_{j}=1$ ). In the MDJ category, a shared function $C$ is learned, e.g., through a neural network. The final predictions are obtained through $\delta_{j}(C(x))$, where $\delta_{j}$ is the function that takes the outcome of $C(x)$ and outputs the prediction probability of the $j$ th disease as well. In this manner, a jointly trained MLL model can predict the probabilities $P_{j}$ (for $1 \leq j \leq J$ ) for all the $J$ diseases simultaneously. See Figure 2 .

\subsection{KAA: Leveraging Biomedical Literature during Multi-label Prediction}

The key idea of KAA is that the predictions for two similar diseases can be fine-tuned based on their similarity obtained from the knowledge graph to reduce the divergence between their predictions. If two diseases are not similar, then we rely primarily on data-driven probabilities. KAA iteratively applies this to all pairs of diseases. Similar iterative procedures have been used in other contexts, e.g., Reference [17].

Let $S_{d, d^{\prime}}$ be the similarity between the knowledge graph embeddings of diseases $d$ and $d^{\prime}$.

To impose the condition that two diseases with high similarity tend to have similar occurrence probabilities, we define the following loss function where the probabilities $P_{j}: j \in J$ represent purely data-driven predictions from any existing algorithm for MDPA and the probabilities $P_{j}^{\prime}, P_{k}^{\prime}$ : $j \in J, k \in J$ represent our proposed knowledge-refined predictions.

$$
f\left(P_{j}^{\prime}\right)=(1-\epsilon) \sum_{j=1}^{J} \sum_{k=1}^{J} S_{j, k}\left(P_{j}^{\prime}-P_{k}^{\prime}\right)^{2}+\epsilon \sum_{j=1}^{J}\left\|S_{j, *}\right\|_{1}\left(P_{j}^{\prime}-P_{j}\right)^{2}
$$

Equation (1) consists of two terms. The first term captures the constraint on disease similarity. For a pair of diseases, $j$ and $k$, with large similarity value $S_{j, k}$, minimizing $f\left(P_{j}^{\prime}\right)$ will lead to smaller difference between the knowledge-refined probabilities $P_{j}^{\prime}$ and $P_{k}^{\prime}$. However, a small similarity value $S_{j, k}$ will not enforce such a constraint on the difference between the knowledge-refined probabilities. The second term is used to ensure that knowledge-corrected probabilities do not deviate too much from the data-driven predictions. This can be viewed as a form of regularization, as we do not want this optimization to completely overrule the model learned during training. Hence, the squared difference $\left(P_{j}^{\prime}-P_{j}\right)^{2}$ is minimized with a coefficient $\left\|S_{j, *}\right\|_{1}=\sum_{k=1}^{J}\left|S_{j, k}\right|$ to ensure that both terms have comparable coefficients. This coefficient prevents the loss function from attaching more importance to the first term over summations involving $P_{j}^{\prime}: j \in J$ when $\left\|S_{j, *}\right\|_{1}$ is large. The tradeoff between the first and second terms in Equation (1) is controlled by a hyperparameter $\epsilon \in(0,1)$. To minimize Equation (1), we find its critical point:

$$
\frac{\partial\left(f\left(P_{j}^{\prime}\right)\right)}{\partial\left(P_{j}^{\prime}\right)} \propto(1-\epsilon) \sum_{j=1}^{J} \sum_{k=1}^{J} S_{j, k}\left(P_{j}^{\prime}-P_{k}^{\prime}\right)+\epsilon \sum_{j=1}^{J}\left\|S_{j, *}\right\|_{1}\left(P_{j}^{\prime}-P_{j}\right) . \quad \forall j \in J
$$


Setting the above to zero, we obtain the refined prediction for disease $j$, which consists of the original prediction $P_{j}$ and a "weighted-average" of optimal $P_{k}^{\prime},(\forall k \in J)$ :

$$
P_{j}^{\prime}=(1-\epsilon) \frac{\sum_{k=1}^{J} S_{j, k}, P_{k}^{\prime}}{\left\|S_{j, *}\right\|_{1}}+\epsilon P_{j}
$$

We note that the second-order partial derivative, shown in Equation (4), is greater than zero for positive similarity values ensuring that the critical point is a minimum.

$$
\frac{\partial^{2}\left(f\left(P_{j}^{\prime}\right)\right)}{\partial^{2}\left(P_{j}^{\prime}\right)}=(1-\epsilon) \sum_{j=1}^{J} \sum_{k=1}^{J} S_{j, k}+\epsilon \sum_{j=1}^{J}\left\|S_{j, *}\right\|_{1}=\sum_{j=1}^{J} \sum_{k=1}^{J} S_{j, k}
$$

We then obtain an iterative process that allows us to input a set of knowledge-refined predictions to obtain another set of updated predictions, as shown in Equation (5). We initialize $P_{j}^{\prime(t=0)}(t$ is the iteration index) with the data-driven predictions $P_{j}$ (i.e., $P_{j}^{\prime(0)}=P_{j}$ ). We repeat this step for all $J$ diseases to obtain $P_{j}^{\prime(t=1)}, 1 \leq j \leq J$ and iteratively continue until convergence.

$$
P_{j}^{\prime(t)}=(1-\epsilon) \frac{\sum_{k=1}^{J} S_{j, k}, P_{k}^{\prime(t-1)}}{\left\|S_{j, *}\right\|_{1}}+\epsilon P_{j}
$$

Convergence is determined by no decrease in loss in successive iterations, which typically occurs in 10-15 iterations in our experiments. The time complexity of obtaining the refined predictions for all $J$ labels, for each patient, is $O\left(J^{2} T\right)$ where $T$ is the total number of iterations.

To calculate disease similarities, we use knowledge graph embeddings from TransE [4] in our experiments. We provide a brief overview of TransE in Appendix B. TransE outputs $k$-dimensional vector representations (where $k$ is a hyperparameter, set to 200 in our experiments) of all the clinical entities in the graph. Thus, we obtain a 200-dimensional vector representation for each disease. The similarity between two disease vectors $d$ and $d^{\prime}$ is computed using the Gaussian kernel, $S_{d, d^{\prime}}=\exp \left(-\gamma\left\|d-d^{\prime}\right\|\right)^{2}$, where exp denotes the exponential function and $\gamma$ is a hyperparameter. This similarity value is always positive, thereby ensuring a minima in Equation (4).

Figure 2 shows a schematic of how our KAA approach can be combined with MLL methods. More details on the classifiers MDS and MDJ used in our experiments are in Appendix D. Appendix E has a summary of all the steps in KAA described above, including how each step is applied in our experiments.

\section{EXPERIMENT SET UP}

In this study, experiments are conducted using de-identified data from the MIMIC-III dataset [27], a publicly available EHR dataset of 46,520 patients, collected from intensive care units of the Beth Israel Deaconess Medical Center in Boston, USA, between 2001 to 2012. To evaluate our method in not only common diseases but also rare diseases, we choose the 20 most common diseases and the 10 rarest diseases from our EHR dataset. The occurrence distribution of the selected diseases in our dataset is given in Table 1 . The imbalance ratio (IR) that reflects the imbalance among diseases (i.e., the rarity of diseases in this dataset) is computed as below [32], where $\left|D_{j}^{+}\right|$and $\left|D_{j}^{-}\right|$ are the number of positive and negative samples, respectively, for disease $j$.

$$
I R_{j}=\frac{\max \left(\left|D_{j}^{+}\right|,\left|D_{j}^{-}\right|\right)}{\min \left(\left|D_{j}^{+}\right|,\left|D_{j}^{-}\right|\right)}
$$

Additional details of the selected diseases are in Appendix F. 
Table 1. Summary of Occurrences of the 30 Selected Diseases (Appendix F Has More Details)

\begin{tabular}{|c|c|c|c|c|c|c|c|}
\hline $\begin{array}{l}\text { Disease } \\
\text { No. }\end{array}$ & $\begin{array}{l}\text { Short } \\
\text { Name }\end{array}$ & $\begin{array}{c}\text { Number } \\
\text { (Percent) of } \\
\text { Occurrences }\end{array}$ & $\begin{array}{l}\text { Imbalance } \\
\text { ratio }\end{array}$ & $\begin{array}{c}\text { Disease } \\
\text { No. }\end{array}$ & $\begin{array}{l}\text { Short } \\
\text { Name }\end{array}$ & $\begin{array}{c}\text { Number } \\
\text { (Percent) of } \\
\text { Occurrences }\end{array}$ & $\begin{array}{c}\text { Imbalance } \\
\text { ratio }\end{array}$ \\
\hline 1 & SEP & $6,925(14.9 \%)$ & 5.7 & 16 & NSD & $6,924(14.9 \%)$ & 5.7 \\
\hline 2 & $\mathrm{ARF}$ & $9,549(20.5 \%)$ & 3.9 & 17 & HVD & $6,627(14.2 \%)$ & 6.0 \\
\hline 3 & $\mathrm{CHF}$ & $\begin{array}{l}10,432 \\
(22.4 \%)\end{array}$ & 3.5 & 18 & ED & $6,374(13.7 \%)$ & 6.3 \\
\hline 4 & PNE & $6,558(14.1 \%)$ & 6.1 & 19 & $\mathrm{HD}^{*}$ & $128(0.3 \%)$ & 362.4 \\
\hline 5 & FED & $\begin{array}{l}12,731 \\
(27.4 \%)\end{array}$ & 2.7 & 20 & $\mathrm{RF}$ & $9,884(21.2 \%)$ & 3.7 \\
\hline 6 & LD & $4,156(8.9 \%)$ & 10.2 & 21 & $\mathrm{MM}^{*}$ & $154(0.3 \%)$ & 301.1 \\
\hline 7 & $\mathrm{HCSH}$ & $4,889(10.5 \%)$ & 8.5 & 22 & CKD & $5,039(10.8 \%)$ & 8.2 \\
\hline 8 & DA & $9,540(20.5 \%)$ & 3.9 & 23 & SLE* & $311(0.7 \%)$ & 148.6 \\
\hline 9 & MD & $4,405(9.5 \%)$ & 9.6 & 24 & $\mathrm{NECS}^{*}$ & $513(1.1 \%)$ & 89.7 \\
\hline 10 & $\mathrm{EH}$ & $\begin{array}{l}17,924 \\
(38.5 \%)\end{array}$ & 1.6 & 25 & $\mathrm{CIBD}^{*}$ & $421(0.9 \%)$ & 109.5 \\
\hline 11 & $\mathrm{CAH}$ & $\begin{array}{l}13,115 \\
(28.2 \%)\end{array}$ & 2.5 & 26 & MOS $^{*}$ & $406(0.9 \%)$ & 113.6 \\
\hline 12 & DWC & $8,064(17.3 \%)$ & 4.8 & 27 & $\mathrm{MS}^{*}$ & $222(0.5 \%)$ & 208.5 \\
\hline 13 & DLM & $\begin{array}{l}12,250 \\
(26.3 \%)\end{array}$ & 2.8 & 28 & $\mathrm{CNS}^{*}$ & $269(0.6 \%)$ & 171.9 \\
\hline 14 & DMC & $3,414(7.3 \%)$ & 12.6 & 29 & LYM $^{*}$ & $214(0.5 \%)$ & 216.4 \\
\hline 15 & $\mathrm{CD}$ & $\begin{array}{l}13,705 \\
(29.5 \%)\end{array}$ & 2.4 & 30 & CRA* $^{*}$ & $185(0.4 \%)$ & 250.5 \\
\hline
\end{tabular}

*indicates rare disease.

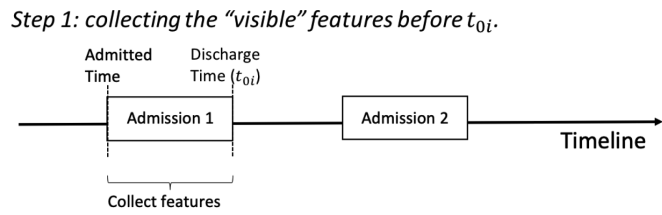

Step 2: predicting the possible diseases in the next $w$ years after $t_{0 i}$

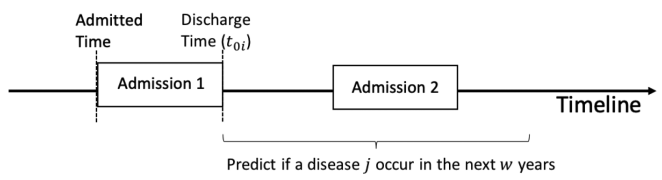

Fig. 3. Our experiment design.

Similar to previous works on MDPA [12,34], we use clinical data from previous admission episodes to predict diagnoses in future episodes, since the granularity of timestamps for many clinical investigations in MIMIC-III is more reliable at an episode-level than at a daily or hourly level. Hence, patients with single episodes are excluded and we use data of 7,537 patients, who have two or more episodes.

Figure 3 illustrates our experiment design. For each patient $i(i=1, \ldots, N)$, we set the discharge time of the first admission as $t_{0 i}$ and use the features collected during the first admission episode as predictors, all of which are the "visible" information for the time after the first admission. We 
Table 2. Disease Occurrences across Time Windows

\begin{tabular}{c|c|ccccc}
\hline Short & Before & \multicolumn{5}{|c}{ During (cumulative) } \\
\cline { 3 - 7 } Name & $t_{o i}$ & Year=w1 & Year=w2 & Year=w3 & Year=w4 & Year=w5 \\
\hline SEP & 1,014 & 1,120 & 1,418 & 1,599 & 1,724 & 1,825 \\
ARF & 1,625 & 1,371 & 1,760 & 2,006 & 2,187 & 2,327 \\
CHF & 2,175 & 1,631 & 2,048 & 2,337 & 2,529 & 2,657 \\
PNE & 1,115 & 969 & 1,236 & 1,391 & 1,513 & 1,599 \\
FED & 2,132 & 1,778 & 2,252 & 2,551 & 2,763 & 2,917 \\
LD & 782 & 606 & 766 & 864 & 932 & 982 \\
HCSH & 983 & 816 & 1,077 & 1,263 & 1,374 & 1,479 \\
DA & 1,720 & 1,433 & 1,824 & 2,068 & 2,228 & 2,354 \\
MD & 727 & 559 & 712 & 809 & 881 & 928 \\
EH & 3,025 & 1,832 & 2,274 & 2,546 & 2,781 & 2,916 \\
CAH & 2,310 & 1,407 & 1,800 & 2,058 & 2,253 & 2,384 \\
DWC & 1,466 & 1,026 & 1,300 & 1,462 & 1,579 & 1,652 \\
DLM & 1,861 & 1,258 & 1,612 & 1,811 & 1,998 & 2,110 \\
DMC & 817 & 543 & 699 & 805 & 886 & 939 \\
CD & 2,348 & 1,740 & 2,148 & 2,407 & 2,586 & 2,730 \\
NSD & 1,053 & 844 & 1,091 & 1,246 & 1,371 & 1,471 \\
HVD & 1,182 & 770 & 979 & 1,113 & 1,211 & 1,283 \\
ED & 1,031 & 801 & 1,041 & 1,194 & 1,309 & 1,390 \\
HD & 30 & 26 & 27 & 29 & 30 & 30 \\
RF & 1,585 & 1,378 & 1,727 & 1,937 & 2,071 & 2,184 \\
MM & 27 & 23 & 28 & 34 & 35 & 39 \\
CKD & 958 & 842 & 1,125 & 1,309 & 1,440 & 1,559 \\
SLE & 88 & 63 & 72 & 81 & 82 & 84 \\
NECS & 75 & 64 & 81 & 95 & 103 & 109 \\
CIBD & 94 & 73 & 93 & 101 & 104 & 111 \\
MOS & 74 & 56 & 76 & 80 & 82 & 85 \\
MS & 50 & 30 & 38 & 45 & 51 & 52 \\
CNS & 48 & 37 & 41 & 44 & 47 & 50 \\
LYM & 39 & 24 & 37 & 38 & 40 & 47 \\
CRA & 36 & 25 & 30 & 34 & 37 & 40 \\
\hline & & & & & & \\
\hline
\end{tabular}

predict the status of $y_{i}^{j}(j=1, \ldots, J)$, which is defined by whether patient $i$ is diagnosed with the $j$ th disease from the label set of $J=30$ diseases in the next $w$ years after $t_{0 i}$. We vary $\mathrm{w}$ from one to five years to examine if different time windows affect our predictive performance.

Table 2 shows the distribution of diseases for different time windows. The first column ("before $t_{0 i}$ ") has the distribution of diseases in the first admission episode, $t_{0 i}$. The second column shows the distribution of diseases that occur within $w=1$ year after $t_{0 i}$. Subsequent columns for $w$ years $(w=2,3,4,5)$ after $t_{0 i}$ shows the distribution of diseases in a cumulative manner.

A total of 5,062 features are extracted from patient demographics, lab tests, procedures, medications, and known diseases. These features are from the hospital record system, which contains measurements for the entire hospital stay, including those in non-ICU care, of these patients [27]. Patient demographics, such as gender, race, marital status, and age are encoded as binary variables (age is binarized into intervals of 10 years, $(0-10, \ldots, 70-80$, and $\geq 80$ ). 
Table 3. Summary of Features Used

\begin{tabular}{ccc}
\hline Feature Category & No. of Columns & Examples \\
\hline Demographics & 76 & Female, married, white \\
Lab tests & 266 & Hematocrit, Eosinophils \\
Procedures & 1,181 & ICD-9-CM procedure codes (e.g., 3727 for cardiac mapping) \\
Medications & 3,271 & "Warfarin, 5mg Tablet" \\
Diagnoses & 268 & ICD-9-CM diagnosis codes (e.g., 5,848 for acute kidney failure) \\
\hline
\end{tabular}

Each medication is encoded as a binary variable indicating whether or not that medication was given. Each lab-test is numerically encoded as two variables (the number of times the test was taken and the number of times the test result was abnormal). Each procedure is encoded as a variable (the number of times the procedure was conducted). The disease diagnosis variables that are known at $t_{0 i}$ are also included as binary predictors. The features used are summarized in Table 3.

We conduct two sets of experiments. In the first set, we compare the improvement in predictive accuracy when KAA is used with multiple MLL models. The selected MLL models include:

(1) Neural network based models for MDS and MDJ (described in Section 2.2).

(2) Classifiers CC-J48 [40] and ML-KNN [53] that were reported to be the best-performing MLL methods on this dataset [57].

The aim of the first experiment is to evaluate whether the use of KAA improves the predictive performance of these MLL models. In addition, we also evaluate two other methods of incorporating general clinical knowledge in MDPA models:

(1) MDJ-Regu [10]: the closest previous approach that uses disease similarities as a regularizer in training MDJ models.

(2) MDS-Embed and MDJ-Embed: Following the common approach of using pretrained embeddings as features, we use KG embeddings of diseases, diagnosed at the first admission as additional inputs in MDS and MDJ.

Since neural networks may overfit such high-dimensional data, we use lasso regularization for MDS and MDJ in all cases, i.e., both with and without knowledge infusion through KAA, knowledge-based regularization (-Regu), and feature embeddings (-Embed). For a fair comparison, we have used exactly the same neural network structure in MDJ, MDJ-Regu, MDJ-Embed, and MDJ-KAA. Similarly, the network structure of MDS, MDS-Embed, and MDS-KAA are identical. We also provide the same disease similarity values, obtained from TransE, to MDJ-Regu, MDJ-Embed, and MDJ-KAA. In MDJ-Embed, multiple diseases, if present in the input diagnoses, are represented by the element-wise sum of the individual disease embeddings. More details of MDS and MDJ networks are in Appendix D.

All the methods are compared using 10-fold cross-validation (CV) to estimate the predictive performance [22]. Since there are multiple labels, we use the micro-AUC, which computes the Area Under the ROC Curve (AUC) by considering the predictions for all the labels together and is preferred when there is class imbalance [50].

In the second set of experiments, we demonstrate the practical impact of KAA by counterfactually analyzing how healthcare predictive models can augment clinicians' capability in identifying high-risk patients and providing preventive treatments to reduce their risks. 
Table 4. Mean Micro-AUC, with Standard Deviations: Prediction of All Diseases across 5 Time Windows

\begin{tabular}{|c|c|c|c|c|c|}
\hline Jindow & w1 & $\mathrm{w} 2$ & w3 & $\mathrm{w} 4$ & w5 \\
\hline MDS & $0.6263 \pm 0.0094$ & $.6293 \pm 0.0034$ & $0.6408 \pm 0.0062$ & $0.6483 \pm 0.0038$ & $0.6496 \pm 0.0054$ \\
\hline MDS-Embed $^{1}$ & $0.6259 \pm 0.0016$ & $0.6419 \pm 0.0033^{* *}$ & $0.6529 \pm 0.0078^{* *}$ & $0.6542 \pm 0.0027^{* *}$ & $0.6521 \pm 0.0045$ \\
\hline IDS-KAA $^{1}$ & $0.6642 \pm 0.0118^{* *}$ & $0.6744 \pm 0.0039^{* *}$ & $0.6850 \pm 0.0041^{* *}$ & $0.6889 \pm 0.0066^{* *}$ & $0.6919 \pm 0.0079^{* *}$ \\
\hline DJ & $0.6885 \pm 0.0192$ & $0.6930 \pm 0.0099$ & $0.7075 \pm 0.0055$ & $0.7157 \pm 0.0116$ & $0.7148 \pm 0.0082$ \\
\hline IDJ-Embed ${ }^{2}$ & $0.6763 \pm 0.0139$ & $0.6827 \pm 0.0136$ & $0.6951 \pm 0.0089$ & $0.6922 \pm 0.0103$ & $0.7051 \pm 0.0087$ \\
\hline 1DJ-Regu ${ }^{2}$ & $0.6704 \pm 0.0173$ & $0.6947 \pm 0.0099$ & $0.7051 \pm 0.0074$ & $0.7175 \pm 0.0090$ & $0.7189 \pm 0.0091$ \\
\hline${\mathrm{MDJ}-\mathrm{KAA}^{2}}^{2}$ & $0.7142 \pm 0.0138^{* *}$ & $0.7261 \pm 0.0094^{* *}$ & $0.7295 \pm 0.0100^{* *}$ & $0.7274 \pm 0.0099^{* *}$ & $0.7348 \pm 0.0063^{* *}$ \\
\hline CC-J48 & $0.7451 \pm 0.0157$ & $0.6333 \pm 0.0061$ & $0.6782 \pm 0.0231$ & $0.6944 \pm 0.0048$ & $0.7041 \pm 0.0025$ \\
\hline CC-J48-KAA ${ }^{3}$ & $0.7746 \pm 0.0165^{* *}$ & $0.6870 \pm 0.0110^{* *}$ & $0.7260 \pm 0.0229^{* *}$ & $0.7349 \pm 0.0049^{* *}$ & $0.7427 \pm 0.0025^{* *}$ \\
\hline ML-KNN & $0.7248 \pm 0.0037$ & $0.7286 \pm 0.0021$ & $0.7326 \pm 0.0013$ & $0.7365 \pm 0.0016$ & $0.7370 \pm 0.0040$ \\
\hline ML-KNN-KAA ${ }^{4}$ & $0.7244 \pm 0.0040$ & $0.7281 \pm 0.0017$ & $0.7320 \pm 0.0013$ & $0.7362 \pm 0.0017$ & $0.7368 \pm 0.0041$ \\
\hline
\end{tabular}

${ }^{* *} p<0.05$. Baseline used for comparison: ${ }^{1} \mathrm{MDS},{ }^{2} \mathrm{MDJ},{ }^{3} \mathrm{CC}-\mathrm{J} 48,{ }^{4} \mathrm{ML}-\mathrm{KNN}$.

Table 5. Mean Micro-AUC, with Standard Deviations: Prediction of Rare Diseases across 5 Time Windows

\begin{tabular}{|c|c|c|c|c|c|}
\hline Time Window & w1 & w2 & w3 & w4 & w5 \\
\hline MDS & $0.5031 \pm 0.0101$ & $0.5088 \pm 0.0044$ & $0.5041 \pm 0.0221$ & $0.5072 \pm 0.0019$ & $0.5095 \pm 0.0016$ \\
\hline MDS-Embed $^{1}$ & $0.5243 \pm 0.0194^{* *}$ & $0.5179 \pm 0.0169$ & $0.5195 \pm 0.0121^{*}$ & $0.5122 \pm 0.0092$ & $0.5161 \pm 0.0099$ \\
\hline MDS-KAA ${ }^{1}$ & $0.5385 \pm 0.0205^{* *}$ & $0.5252 \pm 0.0078^{* *}$ & $0.5253 \pm 0.0178^{* *}$ & $0.5173 \pm 0.0058^{* *}$ & $0.5290 \pm 0.0139^{* *}$ \\
\hline MDJ & $0.6521 \pm 0.0299$ & $0.6464 \pm 0.0169$ & $0.6559 \pm 0.0184$ & $0.6496 \pm 0.0297$ & $0.6607 \pm 0.0149$ \\
\hline MDJ-Embed $^{2}$ & $0.6594 \pm 0.0321$ & $0.6511 \pm 0.0235$ & $0.6567 \pm 0.0222$ & $0.6549 \pm 0.0219$ & $0.6697 \pm 0.0292$ \\
\hline MDJ-Regu ${ }^{2}$ & $0.6560 \pm 0.0438$ & $0.6475 \pm 0.0398$ & $0.6525 \pm 0.0179$ & $0.6550 \pm 0.0355$ & $0.6504 \pm 0.0151$ \\
\hline MDJ-KAA $^{2}$ & $0.6627 \pm 0.0275^{* *}$ & $0.6638 \pm 0.0178^{* *}$ & $0.6704 \pm 0.0197^{* *}$ & $0.6630 \pm 0.0296^{* *}$ & $0.6770 \pm 0.0193^{* *}$ \\
\hline CC-J48 & $0.8249 \pm 0.0379$ & $0.5723 \pm 0.0366$ & $0.6257 \pm 0.0267$ & $0.6361 \pm 0.0393$ & $0.6281 \pm 0.0275$ \\
\hline CC-J48-KAA ${ }^{3}$ & $0.8392 \pm 0.0349^{* *}$ & $0.5897 \pm 0.0282^{* *}$ & $0.6367 \pm 0.0269^{*}$ & $0.6542 \pm 0.0499^{*}$ & $0.6440 \pm 0.0317^{* *}$ \\
\hline ML-KNN & $0.5576 \pm 0.0233$ & $0.5543 \pm 0.0218$ & $0.5564 \pm 0.0350$ & $0.5608 \pm 0.0462$ & $0.5498 \pm 0.0488$ \\
\hline ML-KNN-KAA ${ }^{4}$ & $0.5891 \pm 0.0338^{* *}$ & $0.5888 \pm 0.0305^{* *}$ & $0.5942 \pm 0.0370^{* *}$ & $0.5890 \pm 0.0602^{* *}$ & $0.5868 \pm 0.0491^{* *}$ \\
\hline
\end{tabular}

${ }^{*} p<0.1,{ }^{* *} p<0.05$. Baseline used for comparison: ${ }^{1} \mathrm{MDS},{ }^{2} \mathrm{MDJ},{ }^{3} \mathrm{CC}-\mathrm{J} 48,{ }^{4} \mathrm{ML}-\mathrm{KNN}$.

\section{EXPERIMENTAL RESULTS}

\subsection{Improvement in Predictive Accuracy}

Tables 4 and 5 show the mean and standard deviation (over 10-fold CV) of the micro-AUC obtained by all the methods for all the diseases and the rare diseases, respectively. They are broken down by the length of the time window, $w$. Results at the level of disease labels (i.e., for each of the 30 disease labels) at $w 1$ are presented in Appendix G.

Both MDS-KAA and MDJ-KAA models significantly outperform MDS and MDJ models, respectively, for all five time windows. This shows that the use of KG through KAA indeed improves the predictive performance for all five time windows irrespective of whether multiple diseases are modeled separately or jointly. In addition, we notice that the performance increase from MDS to MDS-KAA model is larger than that from MDJ to MDJ-KAA model. This is understandable, since MDS models do not learn the correlations between diseases during model training, while MDJ models do learn these data-driven correlations due to co-training. Thus, the incremental improvement due to KAA is more for MDS models. More interestingly, our KAA approach improves the performance of MDJ as well where data-driven correlations have been learned by the model during 
training. This clearly illustrates the benefits of additional information from clinical knowledge that is added through KAA for both overall predictive performance and, in particular, for rare diseases.

We observe that the performance values of MDJ and MDJ-Regu model are similar: There is no significant difference in the micro-AUC values in all cases. These results demonstrate that the regularization method employed in MDJ-Regu is not effective in improving predictive performance. Similar findings, that there is limited increase in predictive performance, was reported by Reference [10] when the ICD-9 hierarchy was used to obtain disease similarities in their experiments. Incorporating the knowledge embeddings as inputs, through MDS-Embed, improves the prediction performance of MDS for all diseases as well as rare diseases. However, the improvement in performance from MDS-KAA is higher. In the case of MDJ, using embeddings as inputs through MDJ-Embed does not outperform MDJ-Regu when all diseases are considered. In the case of rare disease, the performance of MDJ-Embed is comparable or marginally better than MDJ-Regu. In both cases, MDJ-KAA outperforms MDJ-Embed. Note that in our experiments, MDJ, MDJ-Embed, MDJ-Regu, and MDJ-KAA use the same co-training model (neural network) and use the same disease similarities obtained from the KG. These results demonstrate that the performance improvement observed is not just due to the use of similarities from KG embeddings but also the way it is used in KAA.

The results for the other classifiers show that KAA improves the performance of CC-J48 for all diseases as well as rare diseases across all five time windows. For ML-KNN, KAA is able to improve the predictive performance in predicting rare diseases, and for all diseases, the performance is comparable to the case without KAA. A paired sample t-test [56] at significance level 0.05 , considering all the time windows over all 10 folds, was conducted to evaluate the significance of performance improvement. We find that performance improvements in rare disease prediction due to the use of KAA are statistically significant for all evaluated MLL models. In the case of all diseases, the use of KAA results in significant improvements over MDS, MDJ, MDJ-Embed, MDJ-Regu, and CC-J48. These results illustrate the generality of our KAA method and performance gain especially for rare diseases.

\subsection{Counterfactual Analysis}

For clinical predictive models, it is vital that "prediction can lead to actions that reduce risk beyond what would occur without the prediction rule" [19]. The practical value of a predictive model is reflected by its ability to facilitate preventive treatments for high-risk patients who otherwise would not get such interventions. Ideally, the impact of a predictive model should be evaluated through a randomized clinical trial on two comparable case and control groups. However, clinical trials are extremely time-consuming and expensive and so, very few clinical predictive models can be evaluated through such trials.

An alternative, proposed by Reference [30], to quantify the utility of a predictive model in improving decision-making, is through a counterfactual analysis. This is done by constructing a counterfactual table, as shown in Figure 4, for each predicted disease (or a group of diseases). We consider all patients who were actually diagnosed with the disease in the period between $t_{i 1}$ and $t_{i 1}+5$ and divide them into four groups. The variables $a, b, c$, and $d$ in the table denote the number of patients in each of the four groups that satisfy the conditions described below.

Such a counterfactual table can offer several insights. The values $a$ and $d$ represent the events that are correctly detected by only the physicians (physician utility) and only our predictive model (model utility), respectively. Values $b$ and $c$ indicate consistency between the physicians' decision and our model's prediction- $b$ : both are correct (positive consistency); $c$ : both are incorrect (negative consistency). Thus, we view $a$ and $c$ as the model's errors (these are the cases where the model predicts "low risk" but the patients are diagnosed with the disease later) and $c$ and $d$ as the 


\begin{tabular}{|c|c|c|c|}
\hline & & Pre & (from a model) \\
\hline & & Low & High \\
\hline Preventive treatment & Yes & $\mathrm{a}$ & $\mathrm{b}$ \\
\hline prescribed at/before $t_{i o}$ & \begin{tabular}{|l|} 
No \\
\end{tabular} & c & $\mathrm{d}$ \\
\hline
\end{tabular}

Fig. 4. Counterfactual table.

physician's limitations (no preventive treatment given to patients who were diagnosed later with the disease). In particular, $c$ should be low, considering that this is the blind area in which both physicians and predictive models cannot recognize the potential risks.

In our case, the most important value is $d$; a higher value indicates greater model utility in enhancing physician's capability. Smaller values of $a$ and $c$ indicate fewer prediction errors and are desirable. To compare two models, a model can be considered more valuable than another if it yields a smaller value for both $a$ and $c$ and a larger value for $d$. Constructing this table requires (1) identifying the cases where physicians recognized future risks (and possibly prescribed preventive interventions) and (2) determining a threshold for distinguishing high and low predicted risks from the considered model. We describe below how they both were determined in our experiments.

EHR data does not have information about whether or not the physician actually recognized the risk of a disease and advised preventive interventions. Moreover, for these 30 diseases, which includes rare diseases, it is difficult for physicians to recognize future risks and prescribe preventive interventions. For a rigorous evaluation of our model, we overestimate the physician's ability by assuming that if there is a known relation in the biomedical literature (as seen in the knowledge graph extracted from the literature), then the physician recognizes it and prescribes the appropriate preventive intervention. No assumptions are made regarding what interventions are provided. The exact nature of the intervention is not required for our evaluation, since we are only evaluating the binary decision of whether or not an intervention was prescribed. The relations considered from the biomedical literature are listed in Appendix $\mathrm{H}$ and include 14 relations such as "predisposes," "precedes," "coexists with," and "causes." So, if a patient has disease A and disease B is known to be related to disease A by any of these 14 relations, then we assume that the physician would prescribe relevant interventions to the patient to prevent disease B. E.g., diabetes (A) is a known to induce hypertension (B), then we assume that preventive intervention (e.g., lifestyle modifications) for disease B is advised.

We consider multiple thresholds $(10 \%, 8 \%$, and $6 \%)$ for distinguishing high and low predicted risks from the predictive model. From previous medical literature, we find that it is common to use $20 \%$ risk over 10 years as a cutoff between high- and low-risk patients (e.g., Reference [29]). Previous works, e.g., References [16, 30], proportionally use $10 \%$ risk cut-off for 5-year span. Hence, in our case considering the 4-year span, we can proportionally use $8 \%$ risk as the cut-off. Once again, we make the evaluation more stringent by using a $10 \%$ threshold. With a $10 \%$ threshold, a patient is considered to be at high-risk of a disease if the predicted probability of the disease is higher than 0.1. Using a lower risk threshold would result in the model predicting more patients at high risk. This would increase the values of $b$ and $d$ in the counterfactual table, thereby showing higher improvement over physician ability. We illustrate this at two other risk thresholds: $6 \%$ and $8 \%$.

Figure 5 presents the aggregated counterfactual results for all the 30 diseases and separately for the 10 rare diseases using risk threshold of $10 \%$ for the predictive models. We compare the results from MDS (MDJ) with the results from MDS-KAA (MDJ-KAA) to illustrate the practical impact 


\begin{tabular}{|c|c|c|c|c|c|c|c|}
\hline \multirow{2}{*}{\multicolumn{2}{|c|}{$\begin{array}{l}\text { Overall diseases } \\
(\# \text { of events }=38333)\end{array}$}} & \multicolumn{2}{|c|}{$\begin{array}{l}\text { Predicted Risk } \\
\text { (MDS) }\end{array}$} & \multirow{4}{*}{$\begin{array}{l}\text { Rare diseases } \\
(\# \text { of events }=647) \\
\text { Prevention } \\
\text { prescribed at } t_{i o}\end{array}$} & & \multicolumn{2}{|c|}{$\begin{array}{l}\text { Predicted Risk } \\
\text { (MDS) }\end{array}$} \\
\hline & & \multirow{2}{*}{\begin{tabular}{|l|} 
Low \\
14140
\end{tabular}} & \multirow{2}{*}{$\begin{array}{l}\text { High } \\
13031 \\
\end{array}$} & & & Low & High \\
\hline \multirow{2}{*}{$\begin{array}{l}\text { Prevention } \\
\text { prescribed at } t_{i o}\end{array}$} & Yes & & & & Yes & 241 & 146 \\
\hline & No & 7158 & 4004 & & No & 187 & 73 \\
\hline \multirow{4}{*}{$\begin{array}{l}\text { Overall diseases } \\
(\# \text { of events }=38333) \\
\text { Prevention } \\
\text { prescribed at } t_{i o}\end{array}$} & & \multicolumn{2}{|c|}{$\begin{array}{l}\text { Predicted Risk } \\
\text { (MDS-KAA) }\end{array}$} & \multirow{4}{*}{$\begin{array}{l}\text { Rare diseases } \\
(\# \text { of events }=647) \\
\quad \text { Prevention } \\
\text { prescribed at } t_{i o}\end{array}$} & & \multicolumn{2}{|c|}{$\begin{array}{l}\text { Predicted Risk } \\
\text { (MDS-KAA) }\end{array}$} \\
\hline & & Low & High & & & Low & High \\
\hline & Yes & 9380 & 17791 & & Yes & 199 & 188 \\
\hline & No & 5629 & 5533 & & No & 173 & 87 \\
\hline \multirow{4}{*}{$\begin{array}{l}\text { Overall diseases } \\
(\# \text { of events }=38333) \\
\text { Prevention } \\
\text { prescribed at } t_{i o}\end{array}$} & & \multicolumn{2}{|c|}{$\begin{array}{l}\text { Predicted Risk } \\
\text { (MDJ) }\end{array}$} & \multirow{5}{*}{$\begin{array}{l}\text { Rare diseases } \\
(\# \text { of events }=647) \\
\text { Prevention } \\
\text { prescribed at } t_{i o}\end{array}$} & & \multicolumn{2}{|c|}{$\begin{array}{l}\text { Predicted Risk } \\
\text { (MDJ) }\end{array}$} \\
\hline & & Low & High & & & Low & High \\
\hline & Yes & 13511 & 13660 & & Yes & 207 & 180 \\
\hline & No & 7225 & 3937 & & No & 190 & 70 \\
\hline \multirow{4}{*}{$\begin{array}{l}\text { Overall diseases } \\
\text { (\# of events }=38333 \text { ) } \\
\text { Prevention } \\
\text { prescribed at } t_{i o}\end{array}$} & & \multicolumn{2}{|c|}{$\begin{array}{c}\text { Predicted Risk } \\
\text { (MDJ-KAA) }\end{array}$} & & & \multicolumn{2}{|c|}{$\begin{array}{c}\text { Predicted Risk } \\
\text { (MDJ-KAA) }\end{array}$} \\
\hline & & Low & High & $\begin{array}{l}\text { Rare diseases } \\
\text { (\# of events }=647 \text { ) }\end{array}$ & & Low & High \\
\hline & Yes & 9175 & 17996 & Prevention & Yes & 176 & 211 \\
\hline & No & 5725 & 5437 & prescribed at $t_{i o}$ & No & 182 & 78 \\
\hline
\end{tabular}

Fig. 5. Results of counterfactual analysis (10\% cut-off).

of incorporating clinical knowledge. The complete results for each of the 30 diseases are shown in Appendix H. Despite the assumed overestimate of physician capability, all values for $\mathrm{d}$ are nonzero, indicating that both MDS and MDJ frameworks provide useful insights for augmenting physicians' decision-making (regardless of diseases, time windows, and models). Notably, both MDS-KAA and MDJ-KAA models that incorporate clinical knowledge outperform the vanilla MDS and MDJ models without knowledge by producing a much smaller value for both $a$ and $c$ (causing fewer errors) and a much larger value for $d$ (augmenting physician's capability). These results demonstrate the efficacy of using our KAA method to leverage clinical knowledge for both common and rare diseases.

Note that our assumptions impose a stringent standard and the results are a conservative underestimate of the model abilities. Since we have overestimated physician ability, the values of $\mathrm{a}$ and $\mathrm{b}$ in our counterfactual tables are expected to be much lower in practice than what we have assumed. Lower values of $\mathrm{a}$ and $\mathrm{b}$ would lead to higher values of $\mathrm{c}$ and $\mathrm{d}$, indicating better model performance compared to the values of $d$ shown now. Moreover, by choosing higher risk thresholds for the predictive models, we underestimate the model capability. The results in Appendix $\mathrm{H}$ for other risk thresholds (8\% and 6\%) show the expected increase in model capability. In particular, for rare diseases, high-risk thresholds should be much lower than $10 \%$. If we reduce the thresholds to below $6 \%$, then we expect the model performance to improve even further. We observe improvements even in this rigorous evaluation regime and expect better performance in less demanding conditions.

\section{DISCUSSION AND IMPLICATIONS}

\subsection{Contributions to the Literature}

This study contributes to existing knowledge base in several ways. First, we design a novel approach to augment MDPA models that can significantly enhance physicians' capabilities for decision-making. MDPA models combined with KAA can capture and utilize correlations between 
diseases from not only data-driven models but also KGs derived from biomedical literature. We validate our KAA approach using real EHR data. Empirical results reveal that the predictive performance of several MDPA models significantly improve with the use of KAA. Overall improvement in predictive performance is seen for all the diseases tested, including rare diseases that are harder to diagnose.

Second, our optimization procedure is flexible and generalizable. It can be combined with any existing MLL approach and can be used with any knowledge graph. Unlike previous approaches that are applied during model training and require modifications in the training process, KAA is applied on the initial predictions obtained from data-driven predictive models. Thus, it can be used with any MDPA model and can easily incorporate the latest knowledge from the biomedical literature without re-training of the MDPA model. In addition, our KAA model does not use a limited subset of biomedical literature (e.g., ICD-9 hierarchy) but associations derived from the entire literature. Moreover, since these associations are automatically mined, the KAA approach can scale well with the fast-growing body of biomedical literature.

Finally, we establish several design principles (i.e., corresponding to the steps in our KAA approach) for MDPA. These design principles can be applied when building an MLL framework to refine the predictions obtained from purely data-driven predictive models through the optimization process using similarities generated from a KG. Such prescriptive knowledge advanced in this study, as "nascent design theory" [21], is generalizable to other contexts of integrating human knowledge with predictive models.

\subsection{Managerial and Practical Implications}

Healthcare resources in hospitals are always limited and have to be utilized effectively for maximum impact on patients' welfare. To do so, hospitals need to not only design the best treatment plans for current conditions of patients but also provide preventive interventions to alleviate potential future problems of patients. Over-investing in the provision of preventive interventions leads to wastage of hospital resources, while under-investing in the provision of preventive interventions potentially increases morbidity rates. Accurate MDPA models allow hospitals to prescribe appropriate levels of preventive interventions for each patient, thereby alleviating the problem of over-investing or under-investing in the provision of preventive interventions. From a patient's perspective, if they are made aware of their risks of various diseases in advance, then they can take actions to reduce the chances of occurrence of these diseases or reduce the severity of their effects, such as through self-management of their daily lives.

Early diagnosis can also lead to significant financial savings. A study revealed that having one additional chronic condition, on average, can cause a patient to incur an additional $\$ 2,000$ in healthcare spending per year [18]. Common diseases (e.g., Diabetes mellitus and Hypertension) have very large patient size and broad societal impact. For instance, as of 2015, 415M people are estimated to have diabetes worldwide [25] and diabetic patients in the United States alone incur a medical cost of $\$ 322 \mathrm{~B}$ per year [8]. Rare diseases are more difficult to identify and may be life-threatening. It can take over seven years, on average, to accurately detect rare diseases [46], leading to prolonged clinical and economic burden.

Although physicians are highly trained professionals in their specific care domain, rare diseases may be overlooked or may be out of the scope of their expertise. Biomedical literature is being created at a rapid rate, and physicians may not have enough time beyond their daily practice to review new knowledge. By leveraging such biomedical literature in a scalable manner, our KAA framework significantly strengthens the capability of predictive systems to offer physicians valuable clinical decision support during the care-giving process. 


\subsection{Limitations and Future Research}

KAA has been evaluated using predictions that were refined using disease similarities using KG embeddings, where other clinical relationships were indirectly modeled. Future studies can consider other relationships (e.g., drug-disease relationships) and extend KAA to directly incorporate these relationships. Our empirical results are obtained based on analyses performed using one EHR dataset. Future work can validate KAA using additional data. Finally, KAA incorporates clinical knowledge after the process of modeling multiple diseases. While there are benefits of such an approach as described earlier, it may be possible to improve the predictive accuracy further by integrating clinical knowledge both during modeling and prediction, which future studies can explore.

\section{CONCLUSION}

This study proposes a novel KAA to enhance data-driven models for MDPA with knowledge from biomedical literature during risk prediction. Our empirical results demonstrate that KAA indeed increases the predictive performance of purely data-driven models for both common and rare diseases. Compared to extant approaches of combined knowledge-based data-driven modelling, KAA offers a more flexible approach, as it can be used in any underlying data-driven MDPA model. This study provides the impetus for further research to leverage the combination of knowledge graphs, which are automatically mined from rapidly growing biomedical literature, and machine learning models, trained on increasingly available EHR data, in creative ways to address complex healthcare challenges. 


\section{APPENDICES}

\section{A SEMMEDDB RELATIONSHIPS}

Tables 6 and 7 lists the relationships found in SemMedDB [28].

Table 6. Summary of Relationships in SemMedDB

\begin{tabular}{ll}
\hline No. & \multicolumn{1}{c}{ Relationship Name: Explanation } \\
\hline 1 & $\begin{array}{l}\text { ADMINISTERED_TO: Given to an entity, when no } \\
\text { assertion is made that the substance or procedure is } \\
\text { being given as treatment. }\end{array}$ \\
2 & $\begin{array}{l}\text { AFFECTS: Produces a direct effect on. Implied here is } \\
\text { the altering or influencing of an existing condition, } \\
\text { state, situation, or entity. }\end{array}$ \\
3 & $\begin{array}{l}\text { ASSOCIATED_WITH: Has a relationship to (gene- } \\
\text { disease relation). }\end{array}$
\end{tabular}

4 AUGMENTS: Expands or stimulates a process.

5 CAUSES: Brings about a condition or an effect. Implied here is that an agent, such as for example, a pharmacologic substance or an organism, has brought about the effect. This includes induces, effects, evokes, and etiology.

6 COEXISTS_WITH: Occurs together with, or jointly.

7 CONVERTS_TO: Changes from one form to another (both substances).

COMPLICATES: Causes to become more severe or complex, or results in adverse effects.

DIAGNOSES: Distinguishes or identifies the nature or characteristics of.

10 DISRUPTS: Alters or influences an already existing condition, state, or situation. Produces a negative effect on.

11 INHIBITS: Decreases, limits, or blocks the action or function of (substance interaction).

13 ISA: The basic hierarchical link in the UMLS Semantic Network. If one item is another item, then the first item is more specific in meaning than the second item.

Food intolerance-related constipation is characterized by proctitis. C0009806: Constipation (Sign or Symptom) - COEX ISTS_WITH - C0033246: Proctitis (Disease or Syndrome)

... plasma nitrite is readily oxidized to nitrate within plasma ... C0028137: Nitrites (Chemical Viewed Structurally) - CONVERTS_TO - C0699857: Nitrate (Inorganic Chemical)

Infections can trigger GBS and exacerbate CIDP. C0021311: Infection (Disease or Syndrome) - COMPLI CATES - C0393819:

Polyradiculoneuropathy, Chronic Inflammatory Demyelinating (Disease or Syndrome)

Manometry showed a higher anal sphincter resting pressure ... C0024751: Manometry (Laboratory Procedure) - DIAG NOSES - C0429217: Anal sphincter pressure (Finding)

Overexpression of NF-kappaB inhibits tumor cell apoptosis. C0079904 NF-kappaB (Amino Acid, Peptide, or Protein) - DISRUPTS - C0162638: Apoptosis (Cell Function)

In recent studies, the BDNF expression was reduced by typical neuroleptics. C0040615: Antipsychotic Agents (Pharmacologic Sub stance) INHIBITS - C0107103: Brain-Derived Neurotrophic Factor (Biologically Active Substance)

Here, we show that chymases, which are chymotryptic peptidases secreted by mast cells, hydrolyze HGF ... C0055673: Chymase (Enzyme) INTERACTS_WITH-C0062534: Hepatocyte Growth Factor (Amino Acid, Peptide, or Protein)

The sympathetic neurotransmitter norepinephrine has been found ... C0028351: Norepinephrine (Neuroreactive Substance or Biogenic Amine) ISA - C0027908: Neurotransmitters (Neuroreactive Substance or Biogenic Amine)

We report a case of primary cardiac epithelioid hemangioendothelioma arising from the right atrium of a 2-month-old infant. C1269890: Entire right atrium (Body Part, Organ, or Organ Component) - LOCATION_OF C0206732: Hemangioendothelioma, Epithelioid (Neoplastic Process)

Recurrence of glomerulopathy underlying ESRD was frequent for IgAN, FSG... C1261469: End stage renal failure (Disease or Syn drome) MANIFESTATION_OF - C1398810: glomerulopathy (Disease or Syndrome) 
Table 7. Summary of Relationships in SemMedDB

\begin{tabular}{ll}
\hline No. & \multicolumn{1}{c}{ Relationship Name: Explanation } \\
\hline 16 & $\begin{array}{l}\text { METHOD_OF: The manner and sequence of events in } \\
\text { performing an act or procedure. }\end{array}$ \\
17 & OCCURS_IN: Has incidence in a group or population.
\end{tabular}

18 PART_OF: Composes, with one or more other physical units, some larger whole. This includes component of, division of, portion of, fragment of, section of, and layer of.

19 PRECEDES: Occurs earlier in time. This includes antedates, comes before, is in advance of, predates, and is prior to.

PREDISPOSES: To be a risk to a disorder, pathology, or condition.

PREVENTS: Stops, hinders or eliminates an action or condition.

PROCESS_OF: Disorder occurs in (higher) organism.

PRODUCES: Brings forth, generates or creates. This includes yields, secretes, emits, biosynthesizes, generates, releases, discharges, and creates.

STIMULATES: Increases or facilitates the action or function of (substance interaction).

TREATS: Applies a remedy with the object of effecting a cure or managing a condition.

USES: Employs in the carrying out of some activity. This includes applies, utilizes, employs, and avails.

COMPARED_WITH: Comparative predicate.

HIGHER_THAN: Comparative predicate.

LOWER_THAN: Comparative predicate.

Examples and Remarks

... because of the use of SSCP as a screening method and sequencing only a part of TSHR exon 10. C0243031: Single-Stranded Conformational Poly morphism (Laboratory or Test Result) - METHOD_OFC0220908:Screening procedure (Health Care Activity)

Older populations are more prone to bone loss with weight loss ... C0599877: loss; bone (Pathologic Function) - OCCUR S_IN - C1518563: Older Population (Human)

The probasal bodies and microtubules within the blepharoplast cavities... C0026046: Microtubules (Cell Component) - PAR T_OF C0230744: Basal body of cilium or flagellum, not bacterial (Cell Component)

... the risk of tissue plasminogen activator-induced hemorrhagic transformation following ischemic stroke in mice ... C0948008: Ischemic stroke (Disease or Syndrome) - PRECEDES - C1096400: Haemorrhagic transformation stroke (Disease or Syndrome)

... high ghrelin levels contribute to obesity in Prader-Willi syndrome (PWS) ... C0911014: ghrelin (Amino Acid, Peptide, or Protein) PREDISPOSES - C0028754: Obesity (Disease or Syndrome)

Ipsapirone and ketanserin protects against circulatory shock, intracranial hypertension, and cerebral ischemia during heatstroke. C0123905: ipsapirone (Pharmacologic Substance) - PRE VENTS C0151740: Intracranial Hypertension (Disease or Syndrome)

... no information is available in CAD patients with normal glomerular filtration rate (GFR). C0010054: Coronary Arteriosclerosis (Disease or Syndrome) - PROCESS_OF - C0030705: Patients (Human)

Human EPCs express functional PAR-1... C0038250: Stem cells (Cell) PRODUCES - C0668084: Receptor, PAR-1 (Amino Acid, Peptide, or Protein)

Candesartan therapy significantly reduced inflammation and increased adiponectin levels ... C0717550: candesartan (Pharmacologic Substance) STIMULATES - C0389071: Adiponectin (Amino Acid, Peptide, or Protein)

This study initially surveyed 163 patients with clinical stage Ib or IIa cervical adenocarcinoma treated with radical hysterectomy and pelvic lymphadenectomy. C0677962: Radical hysterectomy (Therapeutic or Pre ventive Procedure) - TREATS - C0279672: Cervical Adenocarcinoma (Neoplastic Process)

Pre-emptive therapy with oral valganciclovir for CMV infections... C0087111: Therapeutic procedure (Therapeutic or Preventive Procedure) USES - C0909381: valganciclovir (Pharmacologic Substance)

Compared with placebo, candesartan therapy significantly lowered plasma hsCRP levels... C0032042: Placebos (Medical Device) COMPARED_- WITH - C0717550: candesartan (Pharmacologic Substance)

Losartan was more effective than atenolol in reducing cardiovascular morbidity... C0126174: Losartan (Organic Chemical) - HIGH ER_THAN C0004147: Atenolol (Organic Chemical)

Amoxicillin - Clavulanate was not as effective as ciprofloxacin for treating uncomplicated bladder infection in women. C0054066: Amoxicillin-Potassium Clavulanate Combi nation (Antibiotics) LOWER_THAN - C0008809: Ciprofloxacin (Pharmacologic Substance)

Candesartan is as effective as lisinopril once daily in reducing blood pressure. C0717550: candesartan (Organic Chemical) - SAME_AS C0065374: Lisinopril (Amino Acid, Peptide, or Protein) 


\section{B KNOWLEDGE GRAPH EMBEDDING USING TRANSE}

The TransE model is a technical tool used in this study to obtain graph embeddings (i.e., these vector representations containing information on relationships between diseases and other clinical concepts (e.g., drugs)). So, diseases with similar clinical relationships in the knowledge graph will have similar vector representations. These vector representations are further used to obtain similarity between diseases in the augmentation procedure of our KAA approach. The graph in this study is the SemMedDB knowledge graph organized in the form of triples (CUI of disease1, relationship, CUI of disease2), as described above. The embedding obtained through TransE aims to encode global structural knowledge of the graph. We briefly describe the intuition behind TransE and refer the reader to Reference [4] for more details. Given a training set $S$ of triplets $(h, l, t)$ composed of two entities head disease $h$, tail disease $t$, and $h, t \in E$ (the set of clinical concepts), and a relationship $l \in L$ (the set of relationships; head disease has the relationship $l$ to tail disease), TransE model outputs k-dimensional vector representations of the entities and the relationships (where $k$ is a hyperparameter, set to 200 in our experiments). The basic idea behind TransE model is that the relationship induced by the $l$-labeled edges corresponds to a translation of the vector representations. That is, we want that in vector-space $h+l \approx t$ when $(h, l, t)$ holds in the graphs (i.e., head disease $h$ adding the relationship $l$ should be close to tail disease $t$ ). If the triplet $(h, l, t)$ is not present, then $h+l$ should be far away from $t$ in vector space. Following an energy-based framework, the energy of a triplet is equal to $d(h+l, t)$, where $\mathrm{d}$ is a dissimilarity measurement function (e.g., $L 1$ or the $L 2$ norm). To learn the vector representations, a margin-based ranking criterion is minimized over the training set:

$$
L=\sum_{(h, l, t) \in S} \sum_{\left(h^{\prime}, l^{\prime}, t^{\prime}\right) \in S_{(h, l, t)}^{\prime}}\left[\gamma+d(h+l, t)-d\left(h^{\prime}+l, t^{\prime}\right)\right]_{+},
$$

where $[x]_{+}$denotes the positive part of $x, \gamma>0$ denotes a positive margin hyperparameter, and

$$
S_{(h, l, t)}^{\prime}=\left(h^{\prime}, l, t\right)\left|h^{\prime} \notin E \cup\left(h, l, t^{\prime}\right)\right| t^{\prime} \notin E .
$$

The set of corrupted triplets $S^{\prime}$ in Equation (7), consists of training triplets containing either the head diseases or tail diseases replaced by a random entity (but not both at the same time, denoted by $h^{\prime}$ or $t^{\prime}$ ). The loss function (6) favors lower values of the energy for training triplets than for corrupted triplets and thus implement the intended criterion. For a given entity, its vector representation is the same as either the head or the tail of a triplet.

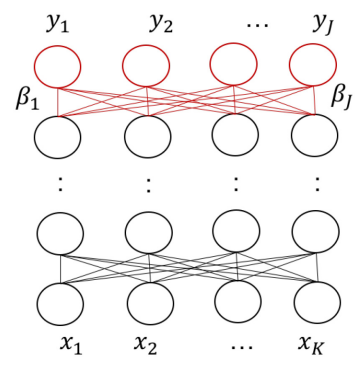

Fig. 6. Regularization of network weights. 


\section{NEURAL NETWORK REGULARIZATION USING DISEASE SIMILARITIES (MDJ-REGU)}

As shown in Figure 6, Reference [10] used a deep feed-forward neural network, with $L$ hidden layers and an output prediction layer for multi-label classification, given $K$ features of a set of samples $X=x_{1}, x_{2}, \ldots, x_{N}$ and the set of labels $Y=y_{1}, y_{2}, \ldots, y_{J}$, where $x_{i} \in R^{K}, y_{i} \in(0,1)^{j},(1 \leq i \leq N)$. We use $\theta=\left(\theta_{\text {hid }}, B\right)$ to denote the model parameters and $\theta_{\text {hid }}=\left(W^{(l)}, b^{(l)}\right)_{(l=1)}^{L}$ denotes the weights for the hidden layers (each with $D^{(l)}$ units) where $D^{(0)}=K$. The $J$ columns of $B=\left[\beta_{1}, \beta_{2}, \ldots, \beta_{J}\right]$ from the last hidden layer are the prediction parameters (where $\beta_{j} \in R^{D^{(L)}}$ ). The neural network consists of fully connected layers, linear activation $\left(W^{(l)} h^{(l-1)}+b^{(l)}\right.$ ) (where $\left.h^{(0)}=x_{i}\right)$ and sigmoid nonlinearities $\left(\delta(z)=1 /(1+\exp (-z))\right.$. The conditional likelihood of $y_{i}$ given $x_{i}$ and model parameters $\theta$ can be written as:

$$
\begin{gathered}
\log P\left(y_{i} \mid x_{i}, \theta\right)=\sum_{j=1}^{J}\left[\epsilon_{j} \log \delta_{j}\left(\beta_{j}^{\top} h_{j}\right)+\left(1-\epsilon_{j}\right) \log \left(1-\delta_{j}\right)\left(\beta_{j}^{\top} h_{j}\right)\right], \\
\operatorname{tr}\left(B^{\top} B\right)=\frac{1}{2} \sum_{1 \leq j, j^{\prime} \leq J} A_{j, j^{\prime}}\left|\beta_{j}-\beta_{j}^{\prime}\right|_{2}^{2},
\end{gathered}
$$

where $\operatorname{tr}($.$) represents the trace operator, the graph Laplacian regularizer enforces the parameters$ $\beta_{j}$ and $\beta_{j^{\prime}}$ to be similar, proportional to $A_{j, j^{\prime}}$. The regularized loss function is:

$$
L=-\sum_{i=1}^{N} \log p\left(y_{i} \mid x_{i}, \theta\right)+\frac{\rho}{2} \operatorname{tr}\left(B^{\top} B\right),
$$

where $r h o>0$ are the Laplacian hyperparameters. Thus, the similarity value in $A_{j, j^{\prime}}$ affects the parameters $\beta_{j}$ and $\beta_{j^{\prime}}$, brings them closer, which in turn makes the predictions for diseases $j, j^{\prime}$ similar.

\section{TRAINING MDS AND MDJ MODELS}

During training, a classifier has to be chosen in both MDS and MDJ frameworks. Any statistical or machine learning method to obtain $J$ binary classifiers $g_{j}$ (for MDS) and co-trained structure $C(x)$ and $\delta_{j},(1 \leq j \leq J)$ (for MDJ) can be adopted. We use a deep neural network (DNN) model with two hidden layers. Hyperparameter tuning can be done on a randomly chosen validation set (that is separated out from the training data). In our experiments, the learning rate, batch size, and two hidden layer sizes for binary classifier $g_{j}$ are set to $0.008,50,100,100$, respectively. The same set of hyperparameters for the co-trained neural network $C$ are set to $0.008,75,2000,100$, and a sigmoid activation function is used for $\delta_{j}$. Number of epochs was set to 100 for MDS and 180 for MDJ. If a binary label is required from the prediction probabilities $\left(P_{j}^{\prime}\right)$, then a threshold probability value has to be chosen: The label is set to 1 above this threshold and to 0 below the threshold. We set the threshold value to a constant a across all labels. This value is chosen empirically to maximize the overall F-measure on the training data [45].

\section{E KAA: ALGORITHM DESCRIPTION}

Table 8 below lists all the steps for using KAA with an MDPA model and a Knowledge Graph.

Hyperparameter $\epsilon$ for KAA and $\gamma$ for the RBF kernel may be selected by grid search by evaluating the performance on a randomly chosen validation set (that is separated out from the training data). In our experiments, we use $\epsilon=0.9, \gamma=0.01$. $\lambda$ can be set to a low value, e.g., 0.0001 ; in 
Table 8. Algorithm for KAA

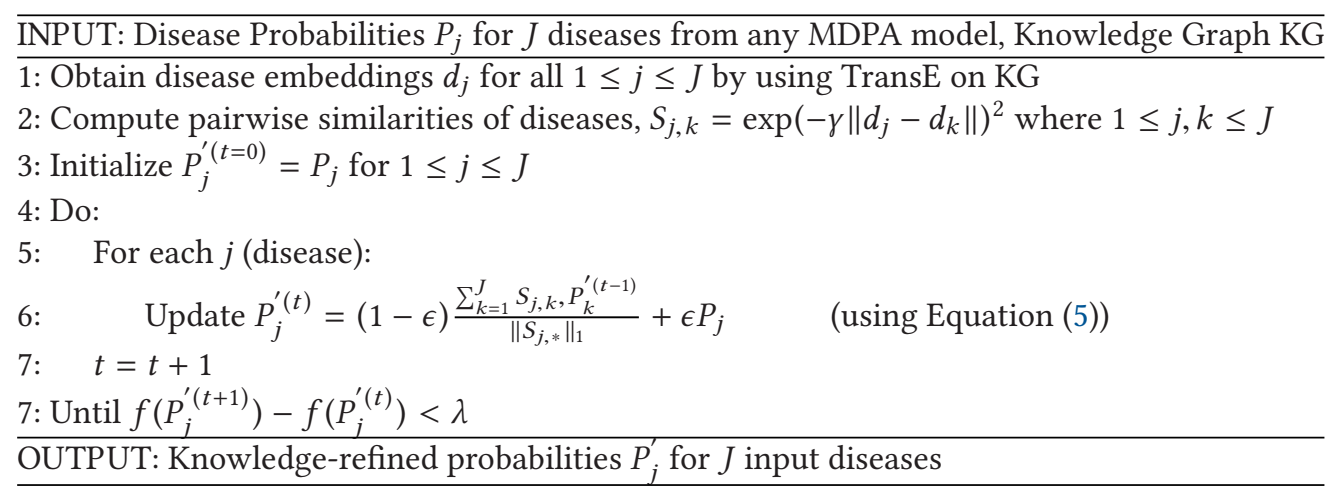

Table 9. Summary of the 30 Selected Diseases

\begin{tabular}{|c|c|c|c|}
\hline No. & Disease Name & Abbreviation & Disease Examples (ICD-9-CM codes) \\
\hline 1 & Septicemia (except in labor) & SEP & Septicemia (038) \\
\hline 2 & Acute and unspecified renal failure & ARF & Kidney Failure, Acute (5849) \\
\hline 3 & Congestive heart failure; nonhypertensive & $\mathrm{CHF}$ & Congestive Heart Failure (4280) \\
\hline 4 & $\begin{array}{l}\text { Pneumonia (except that caused by } \\
\text { tuberculosis or sexually transmitted } \\
\text { disease) }\end{array}$ & PNE & $\begin{array}{l}\text { Pneumonia due to other specified } \\
\text { bacteria (4828) }\end{array}$ \\
\hline 5 & Fluid and electrolyte disorders & FED & $\begin{array}{l}\text { Electrolyte and fluid disorders not } \\
\text { elsewhere classified (2769) }\end{array}$ \\
\hline 6 & Other liver diseases & LD & Non-alcoholic Fatty Liver (5718) \\
\hline 7 & $\begin{array}{l}\text { Hypertension with complications and } \\
\text { secondary hypertension }\end{array}$ & $\mathrm{HCSH}$ & $\begin{array}{l}\text { Cardiovascular Diseases (3062); } \\
\text { Coronary Heart Diseases (402) }\end{array}$ \\
\hline 8 & Deficiency and other anemia & DA & folate-deficiency anemia (2812) \\
\hline 9 & $\begin{array}{l}\text { Other nutritional; endocrine; and } \\
\text { metabolic disorders }\end{array}$ & MD & Obesity (2780) \\
\hline 10 & Essential hypertension & $\mathrm{EH}$ & Essential Hypertension (401) \\
\hline 11 & $\begin{array}{l}\text { Coronary atherosclerosis and other heart } \\
\text { disease }\end{array}$ & $\mathrm{CAH}$ & Coronary Arteriosclerosis (4140) \\
\hline 12 & Diabetes mellitus without complication & DWC & Glucose Intolerance (2713) \\
\hline 13 & Disorders of lipid metabolism & DLM & Hypercholesterolemia $(2720,2722)$ \\
\hline 14 & Diabetes mellitus with complications & $\mathrm{DMC}$ & Diabetic Retinopathy (36201-36207) \\
\hline 15 & Cardiac dysrhythmias & $\mathrm{CD}$ & Cardiac dysrhythmias (427) \\
\hline 16 & Other nervous system disorders & NSD & $\begin{array}{l}\text { Unspecified disorders of nervous } \\
\text { system (3499) }\end{array}$ \\
\hline 17 & Heart valve disorders & HVD & Aortic Valve Stenosis (3960) \\
\hline 18 & Esophageal disorders & $\mathrm{ED}$ & Esophageal hemorrhage (53082) \\
\hline 19 & Hodgkin`s disease & HD & Hodgkin’s disease (201) \\
\hline 20 & $\begin{array}{l}\text { Respiratory failure; insufficiency; arrest } \\
\text { (adult) }\end{array}$ & $\mathrm{RF}$ & Acute respiratory failure (51881) \\
\hline 21 & Multiple myeloma & MM & Multiple myeloma (2030) \\
\hline 22 & Chronic kidney disease & CKD & Chronic kidney disease (585) \\
\hline
\end{tabular}


Table 9. Continued

\begin{tabular}{llll}
\hline No. & \multicolumn{1}{c}{ Disease Name } & Abbreviation & \multicolumn{1}{c}{ Disease Examples (ICD-9-CM codes) } \\
\hline 23 & $\begin{array}{l}\text { Systemic lupus erythematosus and } \\
\text { connective tissue disorders }\end{array}$ & SLE & Systemic lupus erythematosus (7100) \\
24 & Other non-epithelial cancer of skin & NECS & Skin of trunk, except scrotum (1735) \\
25 & Cancer of liver and intrahepatic bile duct & CIBD & Intrahepatic bile ducts (1551) \\
26 & Melanomas of skin & MOS & Malignant melanoma of skin (172) \\
27 & Multiple sclerosis & MS & Multiple sclerosis (340) \\
28 & Other CNS infection and poliomyelitis & CNS & Acute poliomyelitis (045) \\
29 & Lymphadenitis & LYM & Acute lymphadenitis (683) \\
30 & Cancer of rectum and anus & CRA & Malignant neoplasm of rectum, \\
& & & rectosigmoid junction, and anus (154) \\
\hline
\end{tabular}

our experiments, the difference in loss between successive iterations reaches zero within 10-15 iterations.

In our experiments, detailed in Section 4, we have considered 30 diseases (shown in Table 9) from the MIMIC-III clinical database. The Knowledge Graph used is SemMedDB [28]. Table 11 in Appendix $G$ lists the risk probabilities obtained for these 30 diseases at time window 1 from the neural network models MDS and MDJ in columns (1) and (3), respectively. The KAA procedure is applied on these probabilities to obtain knowledge-refined probabilities from MDS-KAA and MDJKAA in columns (2) and (4), respectively. Similarly, KAA is applied on other Multi-label Learning methods and the overall summary of results for all time windows are shown in Tables 4 and 5 .

\section{F DETAILS OF 30 SELECTED DISEASES}

We simultaneously model and predict patients' risks of having 30 diseases (listed in Table 9). These diseases are identified based on the diagnostic definitions from the Health Cost and Utilization Clinical Classification Software (HCUP CCS) [23]. HCUP CCS definitions can cluster ICD-9 diagnostic codes found in EHR data into mutually exclusive, broadly homogeneous disease categories to reduce the noise, ambiguity, and redundancy in the original ICD-9 diagnostic codes. As a result, there are a total of 268 diseases identified from the MIMIC-III dataset.

Table 10. The Summary of the Rarity of Rare Diseases

\begin{tabular}{llc}
\hline Rare diseases name & Frequency & Imbalance ratio \\
\hline Hodgkin's disease & 574,000 & 362.4 \\
Multiple myeloma & 488,200 & 301.1 \\
Systemic lupus erythematosus and & $20-70$ per 100,000 & 148.6 \\
connective tissue disorders & & \\
Other non-epithelial cancer of skin & 392,000 & 89.7 \\
Cancer of liver and intrahepatic bile duct & $1-2$ per 100,000 & 109.5 \\
Melanomas of skin & $3,100,000$ & 113.6 \\
Multiple sclerosis & $2,000,000$ & 208.5 \\
Other CNS infection and poliomyelitis & - & 171.9 \\
Lymphadenitis & - & 216.4 \\
Cancer of rectum and anus & $3,000,000$ & 250.5 \\
\hline
\end{tabular}




\section{Rare Diseases}

To validate the the rarity of the selected rare diseases, we present their frequency (i.e., the proportion of world population affected by these diseases in 2015) $[6,15,41]$ and their imbalance ratios calculated from our dataset, as shown in Table 10. Any disease, disorder, illness, or condition affecting fewer than 200,000 people in the United States is regarded rare by the National Institutes of Health (NIH) and the U.S. Food and Drug Administration (FDA) [9]. To generalize this definition to the rest of the world, we compute the prevalence proportion as $6.23 \times 10^{-4}$ using the estimated population of the U.S. as $321 \mathrm{M}$ [7]. Using this proportion and the estimated world population of 7.358B [37], we get the definition of rare disease to be less than either 4,580,000, or 63 per 100,000 in the world. As shown in Table 3, we find that the diseases selected from our dataset are rare by this definition. The frequency of two diseases- "Other CNS infection and poliomyelitis" and "Lymphadenitis"-could not be found, but both of them should be rare, too, according to their high imbalance ratios.

\section{G RESULTS OF INDIVIDUAL DISEASES}

Table 11 shows the results for individual diseases.

Table 11. Results of Individual Disease at w1

\begin{tabular}{cccccc}
\hline NO. & Disease name & $\begin{array}{c}\text { MDS } \\
(1)\end{array}$ & $\begin{array}{c}\text { MDS-KAA } \\
(2)\end{array}$ & $\begin{array}{c}\text { MDJ } \\
(3)\end{array}$ & $\begin{array}{c}\text { MDJ-KAA } \\
(4)\end{array}$ \\
\hline 1 & SEP & 0.5144 & 0.5494 & 0.5357 & 0.5498 \\
2 & ARF & 0.5503 & 0.5804 & 0.5766 & 0.5920 \\
3 & CHF & 0.5815 & 0.6158 & 0.6398 & 0.6472 \\
4 & PNE & 0.5053 & 0.5309 & 0.5179 & 0.5464 \\
5 & FED & 0.5161 & 0.5161 & 0.5704 & 0.5704 \\
6 & LD & 0.5757 & 0.5861 & 0.6247 & 0.6365 \\
7 & HCSH & 0.5782 & 0.5782 & 0.6654 & 0.6654 \\
8 & DA & 0.5236 & 0.5427 & 0.5452 & 0.5603 \\
9 & MD & 0.5350 & 0.5358 & 0.5613 & 0.5887 \\
10 & EH & 0.5491 & 0.5491 & 0.5773 & 0.5783 \\
11 & CAH & 0.5817 & 0.5770 & 0.6633 & 0.6681 \\
12 & DWC & 0.5471 & 0.5545 & 0.6476 & 0.6509 \\
13 & DLM & 0.5772 & 0.5772 & 0.6039 & 0.6039 \\
14 & DMC & 0.5769 & 0.5771 & 0.6825 & 0.6758 \\
15 & CD & 0.5729 & 0.5953 & 0.6078 & 0.6204 \\
16 & NSD & 0.5396 & 0.5291 & 0.5899 & 0.5922 \\
17 & HVD & 0.5661 & 0.5867 & 0.6187 & 0.6264 \\
18 & ED & 0.5610 & 0.5610 & 0.5920 & 0.5920 \\
19 & HD & 0.4876 & 0.4876 & 0.7684 & 0.7684 \\
20 & RF & 0.5293 & 0.5546 & 0.5696 & 0.5875 \\
21 & MM & 0.4896 & 0.7449 & 0.5570 & 0.7066 \\
22 & CKD & 0.6265 & 0.6570 & 0.6982 & 0.7064 \\
23 & SLE & 0.5271 & 0.6267 & 0.6717 & 0.6897 \\
24 & NECS & 0.5003 & 0.5003 & 0.5701 & 0.5701 \\
25 & CIBD & 0.5256 & 0.5256 & 0.6734 & 0.6831 \\
26 & MOS & 0.5127 & 0.5127 & 0.6648 & 0.6648 \\
\hline & & & & & $($ Continued $)$ \\
& & & & &
\end{tabular}


Table 11. Continued

\begin{tabular}{lccccc}
\hline NO. & Disease name & $\begin{array}{c}\text { MDS } \\
(1)\end{array}$ & $\begin{array}{c}\text { MDS-KAA } \\
(2)\end{array}$ & $\begin{array}{c}\text { MDJ } \\
(3)\end{array}$ & $\begin{array}{c}\text { MDJ-KAA } \\
(4)\end{array}$ \\
\hline 27 & MS & 0.5209 & 0.5209 & 0.8261 & 0.8419 \\
28 & CNS & 0.4882 & 0.4882 & 0.6263 & 0.6263 \\
29 & LYM & 0.4882 & 0.4882 & 0.5146 & 0.5146 \\
30 & CRA & 0.4909 & 0.4909 & 0.5713 & 0.5713 \\
\hline
\end{tabular}

\section{H COUNTERFACTUAL ANALYSIS}

Table 12 lists the positive associations in SemMedDB considered as triggers for diseases.

Table 12. Summary of Positive Relationships between Diseases

\begin{tabular}{|c|c|}
\hline Relationship & Explanation \\
\hline AFFECTS & Produces a direct effect on \\
\hline AUGMENTS & Expands or stimulates a process \\
\hline CAUSES & Brings about a condition or an effect \\
\hline COEXISTS_WITH & Occurs together with, or jointly \\
\hline CONVERTS_TO & Changes from one form to another \\
\hline COMPLICATES & Causes to become more severe or complex, or results in adverse effects \\
\hline ISA & The first item is more specific in meaning than the second item \\
\hline MANIFESTATION_OF & That part of a phenomenon that is directly observable \\
\hline OCCURS_IN & Has incidence in a group \\
\hline PRECEDES & Occurs earlier in time \\
\hline PREDISPOSES & To be a risk to a disorder, pathology, or condition \\
\hline PRODUCES & Brings forth, generates or creates \\
\hline STIMULATES & Increases or facilitates the action or function of \\
\hline SAME_AS & Comparative predicate \\
\hline
\end{tabular}

Figures 7 and 8 show the results at $8 \%$ and $6 \%$ cut-off values to distinguish between high-risk and low-risk based on model predictions. 


\begin{tabular}{|c|c|c|c|c|c|c|c|}
\hline \multirow{2}{*}{\multicolumn{2}{|c|}{$\begin{array}{l}\text { Overall diseases } \\
(\# \text { of events }=38333)\end{array}$}} & \multicolumn{2}{|c|}{$\begin{array}{l}\text { Predicted Risk } \\
\text { (MDS) }\end{array}$} & \multirow{4}{*}{$\begin{array}{l}\text { Rare diseases } \\
\text { (\# of events }=647 \text { ) } \\
\text { Prevention } \\
\text { prescribed at } t_{i o}\end{array}$} & & \multicolumn{2}{|c|}{$\begin{array}{l}\text { Predicted Risk } \\
\text { (MDS) }\end{array}$} \\
\hline & & \multirow{2}{*}{$\begin{array}{l}\text { Low } \\
14140 \\
\end{array}$} & \multirow{2}{*}{$\begin{array}{l}\text { High } \\
13031 \\
\end{array}$} & & & Low & High \\
\hline \multirow{2}{*}{$\begin{array}{l}\text { Prevention } \\
\text { prescribed at } t_{i o}\end{array}$} & Yes & & & & Yes & 241 & 146 \\
\hline & No & 7158 & 4004 & & No & 187 & 73 \\
\hline \multirow{4}{*}{$\begin{array}{l}\text { Overall diseases } \\
(\# \text { of events }=38333) \\
\text { Prevention } \\
\text { prescribed at } t_{i o}\end{array}$} & & \multicolumn{2}{|c|}{$\begin{array}{c}\text { Predicted Risk } \\
\text { (MDS-KAA) }\end{array}$} & \multirow{4}{*}{$\begin{array}{l}\text { Rare diseases } \\
(\# \text { of events }=647) \\
\text { Prevention } \\
\text { prescribed at } t_{i o}\end{array}$} & & \multicolumn{2}{|c|}{$\begin{array}{l}\text { Predicted Risk } \\
\text { (MDS-KAA) }\end{array}$} \\
\hline & & Low & High & & & Low & High \\
\hline & Yes & 8645 & 18526 & & Yes & 190 & 197 \\
\hline & No & 5392 & 5770 & & No & 172 & 88 \\
\hline \multirow{4}{*}{$\begin{array}{l}\text { Overall diseases } \\
(\# \text { of events }=38333) \\
\text { Prevention } \\
\text { prescribed at } t_{i o}\end{array}$} & & \multicolumn{2}{|c|}{$\begin{array}{l}\text { Predicted Risk } \\
\text { (MDJ) }\end{array}$} & \multirow{5}{*}{$\begin{array}{l}\text { Rare diseases } \\
(\# \text { of events }=647) \\
\text { Prevention } \\
\text { prescribed at } t_{i o}\end{array}$} & & \multicolumn{2}{|c|}{$\begin{array}{l}\text { Predicted Risk } \\
\text { (MDJ) }\end{array}$} \\
\hline & & Low & High & & & Low & High \\
\hline & Yes & 13506 & 13665 & & Yes & 207 & 180 \\
\hline & No & 7225 & 3937 & & No & 190 & 70 \\
\hline \multirow{4}{*}{$\begin{array}{l}\text { Overall diseases } \\
(\# \text { of events }=38333) \\
\text { Prevention } \\
\text { prescribed at } t_{i o}\end{array}$} & & \multicolumn{2}{|c|}{$\begin{array}{c}\text { Predicted Risk } \\
\text { (MDJ-KAA) }\end{array}$} & & & \multicolumn{2}{|c|}{$\begin{array}{l}\text { Predicted Risk } \\
\text { (MDJ-KAA) }\end{array}$} \\
\hline & & Low & High & (\# of events $=647$ ) & & Low & High \\
\hline & Yes & 8584 & 18587 & Prevention & Yes & 173 & 214 \\
\hline & No & 5478 & 5684 & prescribed at $t_{i o}$ & No & 180 & 80 \\
\hline
\end{tabular}

Fig. 7. Results of counterfactual analysis ( $8 \%$ cut-off).

\begin{tabular}{|c|c|c|c|c|c|c|c|}
\hline \multirow{2}{*}{\multicolumn{2}{|c|}{$\begin{array}{l}\text { Overall diseases } \\
(\# \text { of events }=38333)\end{array}$}} & \multicolumn{2}{|c|}{$\begin{array}{l}\text { Predicted Risk } \\
\text { (MDS) }\end{array}$} & \multirow{4}{*}{$\begin{array}{l}\text { Rare diseases } \\
(\# \text { of events }=647) \\
\text { Prevention } \\
\text { prescribed at } t_{i o}\end{array}$} & & \multicolumn{2}{|c|}{$\begin{array}{l}\text { Predicted Risk } \\
\text { (MDS) }\end{array}$} \\
\hline & & \multirow{2}{*}{$\begin{array}{l}\text { Low } \\
14138 \\
\end{array}$} & \multirow{2}{*}{$\begin{array}{l}\text { High } \\
13033 \\
\end{array}$} & & & Low & High \\
\hline \multirow{2}{*}{$\begin{array}{l}\text { Prevention } \\
\text { prescribed at } t_{i o}\end{array}$} & Yes & & & & Yes & 241 & 146 \\
\hline & No & 7158 & 4004 & & No & 187 & 73 \\
\hline \multirow{4}{*}{$\begin{array}{l}\text { Overall diseases } \\
(\# \text { of events }=38333) \\
\text { Prevention } \\
\text { prescribed at } t_{i o}\end{array}$} & & \multicolumn{2}{|c|}{$\begin{array}{c}\text { Predicted Risk } \\
\text { (MDS-KAA) }\end{array}$} & \multirow{4}{*}{$\begin{array}{l}\text { Rare diseases } \\
(\# \text { of events }=647 \text { ) } \\
\text { Prevention } \\
\text { prescribed at } t_{i o}\end{array}$} & & \multicolumn{2}{|c|}{$\begin{array}{l}\text { Predicted Risk } \\
\text { (MDS-KAA) }\end{array}$} \\
\hline & & Low & High & & & Low & High \\
\hline & Yes & 7858 & 19313 & & Yes & 179 & 208 \\
\hline & No & 5027 & 6135 & & No & 167 & 93 \\
\hline \multirow{4}{*}{$\begin{array}{l}\text { Overall diseases } \\
(\# \text { of events }=38333) \\
\text { Prevention } \\
\text { prescribed at } t_{i o}\end{array}$} & & \multicolumn{2}{|c|}{$\begin{array}{l}\text { Predicted Risk } \\
\text { (MDJ) }\end{array}$} & \multirow{5}{*}{$\begin{array}{l}\text { Rare diseases } \\
(\# \text { of events }=647 \text { ) } \\
\text { Prevention } \\
\text { prescribed at } t_{i o}\end{array}$} & & \multicolumn{2}{|c|}{$\begin{array}{l}\text { Predicted Risk } \\
\text { (MDJ) }\end{array}$} \\
\hline & & Low & High & & & Low & High \\
\hline & Yes & 13511 & 13660 & & Yes & 207 & 180 \\
\hline & No & 7225 & 3937 & & No & 190 & 70 \\
\hline \multirow{4}{*}{$\begin{array}{l}\text { Overall diseases } \\
(\# \text { of events }=38333) \\
\text { Prevention } \\
\text { prescribed at } t_{i o}\end{array}$} & & \multicolumn{2}{|c|}{$\begin{array}{c}\text { Predicted Risk } \\
\text { (MDJ-KAA) }\end{array}$} & & & \multicolumn{2}{|c|}{$\begin{array}{l}\text { Predicted Risk } \\
\text { (MDJ-KAA) }\end{array}$} \\
\hline & & Low & High & \multirow{3}{*}{$\begin{array}{l}\text { Rare diseases } \\
(\# \text { of events }=647) \\
\text { Prevention } \\
\text { prescribed at } t_{i o}\end{array}$} & & Low & High \\
\hline & Yes & 9175 & 17996 & & Yes & 164 & 223 \\
\hline & No & 5725 & 5437 & & No & 178 & 82 \\
\hline
\end{tabular}

Fig. 8. Results of counterfactual analysis (6\% cut-off). 
The results for individual diseases with MDS model are shown in Tables 13, 14, and 15 for cut-off values $10 \%, 8 \%$, and $6 \%$, respectively.

Table 13. Results of Counterfactual Analyses for MDS (10\% Cut-off)

\begin{tabular}{|c|c|c|c|c|c|c|c|c|}
\hline \multirow{2}{*}{$\begin{array}{c}\text { Disease } \\
\text { name }\end{array}$} & \multicolumn{4}{|c|}{ MDS-not-KAA } & \multicolumn{4}{|c|}{ MDS-KAA } \\
\hline & $\mathrm{a}$ & $\mathrm{b}$ & $\mathrm{c}$ & $\mathrm{d}$ & $\mathrm{a}$ & $\mathrm{b}$ & $\mathrm{c}$ & $\mathrm{d}$ \\
\hline SEP & 932 & 726 & 105 & 62 & 492 & 1,166 & 69 & 98 \\
\hline $\mathrm{ARF}$ & 1,217 & 980 & 85 & 45 & 719 & 1,478 & 61 & 69 \\
\hline $\mathrm{CHF}$ & 1,268 & 1,285 & 67 & 37 & 768 & 1,785 & 41 & 63 \\
\hline PNE & 767 & 567 & 177 & 88 & 416 & 918 & 116 & 149 \\
\hline FED & 516 & 588 & 1,072 & 741 & 516 & 588 & 1,072 & 741 \\
\hline LD & 468 & 369 & 100 & 45 & 255 & 582 & 57 & 88 \\
\hline $\mathrm{HCSH}$ & 388 & 335 & 524 & 232 & 388 & 335 & 524 & 232 \\
\hline DA & 1,101 & 962 & 178 & 113 & 630 & 1,433 & 125 & 166 \\
\hline MD & 208 & 190 & 348 & 182 & 116 & 282 & 210 & 320 \\
\hline $\mathrm{EH}$ & 1,229 & 1,298 & 217 & 172 & 994 & 1,533 & 169 & 220 \\
\hline $\mathrm{CAH}$ & 840 & 889 & 416 & 239 & 523 & 1,206 & 253 & 402 \\
\hline DWC & 865 & 671 & 67 & 49 & 485 & 1,051 & 39 & 77 \\
\hline DLM & 588 & 619 & 574 & 329 & 588 & 619 & 574 & 329 \\
\hline DMC & 415 & 305 & 157 & 62 & 415 & 305 & 157 & 62 \\
\hline $\mathrm{CD}$ & 994 & 1,137 & 354 & 245 & 568 & 1,563 & 232 & 367 \\
\hline NSD & 574 & 421 & 349 & 127 & 323 & 672 & 220 & 256 \\
\hline HVD & 466 & 375 & 306 & 136 & 257 & 584 & 171 & 271 \\
\hline ED & 310 & 311 & 505 & 264 & 310 & 311 & 505 & 264 \\
\hline HD & 15 & 9 & 4 & 2 & 15 & 9 & 4 & 2 \\
\hline $\mathrm{RF}$ & 387 & 457 & 793 & 547 & 208 & 636 & 473 & 867 \\
\hline MM & 16 & 8 & 12 & 3 & 11 & 13 & 9 & 6 \\
\hline CKD & 366 & 400 & 577 & 216 & 210 & 556 & 388 & 405 \\
\hline SLE & 43 & 27 & 8 & 6 & 16 & 54 & 3 & 11 \\
\hline NECS & 19 & 12 & 55 & 23 & 19 & 12 & 55 & 23 \\
\hline CIBD & 42 & 30 & 27 & 12 & 34 & 38 & 21 & 18 \\
\hline MOS & 39 & 21 & 18 & 7 & 39 & 21 & 18 & 7 \\
\hline MS & 32 & 12 & 6 & 2 & 30 & 14 & 6 & 2 \\
\hline CNS & 21 & 13 & 9 & 7 & 21 & 13 & 9 & 7 \\
\hline LYM & 2 & 3 & 34 & 8 & 2 & 3 & 34 & 8 \\
\hline CRA & 12 & 11 & 14 & 3 & 12 & 11 & 14 & 3 \\
\hline Overall Diseases & 14,140 & 13,031 & 7,158 & 4,004 & 9,380 & 17,791 & 5,629 & 5,533 \\
\hline Rare Diseases & 241 & 146 & 187 & 73 & 199 & 188 & 173 & 87 \\
\hline
\end{tabular}


Table 14. Results of Counterfactual Analyses for MDS (8\% Cut-off)

\begin{tabular}{|c|c|c|c|c|c|c|c|c|}
\hline \multirow{2}{*}{$\begin{array}{c}\text { Disease } \\
\text { name }\end{array}$} & \multicolumn{4}{|c|}{ MDS-not-KAA } & \multicolumn{4}{|c|}{ MDS-KAA } \\
\hline & $\mathrm{a}$ & $\mathrm{b}$ & $\mathrm{c}$ & $\mathrm{d}$ & $\mathrm{a}$ & $\mathrm{b}$ & $\mathrm{c}$ & $\mathrm{d}$ \\
\hline SEP & 932 & 726 & 105 & 62 & 424 & 1,234 & 63 & 104 \\
\hline ARF & 1,217 & 980 & 85 & 45 & 622 & 1,575 & 57 & 73 \\
\hline $\mathrm{CHF}$ & 1,268 & 1,285 & 67 & 37 & 675 & 1,878 & 39 & 65 \\
\hline PNE & 767 & 567 & 177 & 88 & 355 & 979 & 104 & 161 \\
\hline FED & 516 & 588 & 1,072 & 741 & 516 & 588 & 1,072 & 741 \\
\hline LD & 468 & 369 & 100 & 45 & 229 & 608 & 53 & 92 \\
\hline $\mathrm{HCSH}$ & 388 & 335 & 524 & 232 & 388 & 335 & 524 & 232 \\
\hline DA & 1,101 & 962 & 178 & 113 & 544 & 1,519 & 104 & 187 \\
\hline MD & 208 & 190 & 348 & 182 & 96 & 302 & 177 & 353 \\
\hline $\mathrm{EH}$ & 1,229 & 1,298 & 217 & 172 & 944 & 1,583 & 164 & 225 \\
\hline $\mathrm{CAH}$ & 840 & 889 & 416 & 239 & 500 & 1,229 & 229 & 426 \\
\hline DWC & 865 & 671 & 67 & 49 & 424 & 1,112 & 36 & 80 \\
\hline DLM & 588 & 619 & 574 & 329 & 588 & 619 & 574 & 329 \\
\hline DMC & 415 & 305 & 157 & 62 & 415 & 305 & 157 & 62 \\
\hline $\mathrm{CD}$ & 994 & 1,137 & 354 & 245 & 538 & 1,593 & 224 & 375 \\
\hline NSD & 574 & 421 & 349 & 127 & 277 & 718 & 191 & 285 \\
\hline HVD & 466 & 375 & 306 & 136 & 236 & 605 & 163 & 279 \\
\hline ED & 310 & 311 & 505 & 264 & 310 & 311 & 505 & 264 \\
\hline HD & 15 & 9 & 4 & 2 & 15 & 9 & 4 & 2 \\
\hline $\mathrm{RF}$ & 387 & 457 & 793 & 547 & 185 & 659 & 427 & 913 \\
\hline MM & 16 & 8 & 12 & 3 & 8 & 16 & 9 & 6 \\
\hline CKD & 366 & 400 & 577 & 216 & 189 & 577 & 357 & 436 \\
\hline SLE & 43 & 27 & 8 & 6 & 15 & 55 & 3 & 11 \\
\hline NECS & 19 & 12 & 55 & 23 & 19 & 12 & 55 & 23 \\
\hline CIBD & 42 & 30 & 27 & 12 & 32 & 40 & 20 & 19 \\
\hline MOS & 39 & 21 & 18 & 7 & 39 & 21 & 18 & 7 \\
\hline MS & 32 & 12 & 6 & 2 & 27 & 17 & 6 & 2 \\
\hline CNS & 21 & 13 & 9 & 7 & 21 & 13 & 9 & 7 \\
\hline LYM & 2 & 3 & 34 & 8 & 2 & 3 & 34 & 8 \\
\hline CRA & 12 & 11 & 14 & 3 & 12 & 11 & 14 & 3 \\
\hline Overall Diseases & 14,140 & 13,031 & 7,158 & 4,004 & 8,645 & 18,526 & 5,392 & 5,770 \\
\hline Rare Diseases & 241 & 146 & 187 & 73 & 190 & 197 & 172 & 88 \\
\hline
\end{tabular}


Table 15. Results of Counterfactual Analyses for MDS (6\% Cut-off)

\begin{tabular}{|c|c|c|c|c|c|c|c|c|}
\hline \multirow{2}{*}{$\begin{array}{c}\text { Disease } \\
\text { name }\end{array}$} & \multicolumn{4}{|c|}{ MDS-not-KAA } & \multicolumn{4}{|c|}{ MDS-KAA } \\
\hline & $\mathrm{a}$ & $\mathrm{b}$ & $\mathrm{c}$ & $\mathrm{d}$ & $\mathrm{a}$ & $\mathrm{b}$ & $\mathrm{c}$ & $\mathrm{d}$ \\
\hline SEP & 931 & 727 & 105 & 62 & 382 & 1,276 & 54 & 113 \\
\hline ARF & 1,217 & 980 & 85 & 45 & 573 & 1,624 & 53 & 77 \\
\hline $\mathrm{CHF}$ & 1,267 & 1,286 & 67 & 37 & 625 & 1,928 & 36 & 68 \\
\hline PNE & 767 & 567 & 177 & 88 & 315 & 1,019 & 96 & 169 \\
\hline FED & 516 & 588 & 1,072 & 741 & 516 & 588 & 1,072 & 741 \\
\hline LD & 468 & 369 & 100 & 45 & 171 & 666 & 46 & 99 \\
\hline $\mathrm{HCSH}$ & 388 & 335 & 524 & 232 & 388 & 335 & 524 & 232 \\
\hline DA & 1,101 & 962 & 178 & 113 & 491 & 1,572 & 96 & 195 \\
\hline MD & 208 & 190 & 348 & 182 & 82 & 316 & 141 & 389 \\
\hline $\mathrm{EH}$ & 1,229 & 1,298 & 217 & 172 & 843 & 1,684 & 149 & 240 \\
\hline $\mathrm{CAH}$ & 840 & 889 & 416 & 239 & 459 & 1,270 & 208 & 447 \\
\hline DWC & 865 & 671 & 67 & 49 & 378 & 1,158 & 33 & 83 \\
\hline DLM & 588 & 619 & 574 & 329 & 588 & 619 & 574 & 329 \\
\hline DMC & 415 & 305 & 157 & 62 & 415 & 305 & 157 & 62 \\
\hline$C D$ & 994 & 1,137 & 354 & 245 & 432 & 1,699 & 176 & 423 \\
\hline NSD & 574 & 421 & 349 & 127 & 209 & 786 & 154 & 322 \\
\hline HVD & 466 & 375 & 306 & 136 & 199 & 642 & 137 & 305 \\
\hline ED & 310 & 311 & 505 & 264 & 310 & 311 & 505 & 264 \\
\hline HD & 15 & 9 & 4 & 2 & 15 & 9 & 4 & 2 \\
\hline $\mathrm{RF}$ & 387 & 457 & 793 & 547 & 139 & 705 & 346 & 994 \\
\hline MM & 16 & 8 & 12 & 3 & 4 & 20 & 8 & 7 \\
\hline CKD & 366 & 400 & 577 & 216 & 164 & 602 & 303 & 490 \\
\hline SLE & 43 & 27 & 8 & 6 & 14 & 56 & 2 & 12 \\
\hline NECS & 19 & 12 & 55 & 23 & 19 & 12 & 55 & 23 \\
\hline CIBD & 42 & 30 & 27 & 12 & 31 & 41 & 19 & 20 \\
\hline MOS & 39 & 21 & 18 & 7 & 39 & 21 & 18 & 7 \\
\hline MS & 32 & 12 & 6 & 2 & 22 & 22 & 4 & 4 \\
\hline CNS & 21 & 13 & 9 & 7 & 21 & 13 & 9 & 7 \\
\hline LYM & 2 & 3 & 34 & 8 & 2 & 3 & 34 & 8 \\
\hline CRA & 12 & 11 & 14 & 3 & 12 & 11 & 14 & 3 \\
\hline Overall Diseases & 14,138 & 13,033 & 7,158 & 4,004 & 7,858 & 19,313 & 5,027 & 6,135 \\
\hline Rare Diseases & 241 & 146 & 187 & 73 & 179 & 208 & 167 & 93 \\
\hline
\end{tabular}


The results for individual diseases with MDJ model are shown in Tables 16, 17, and 18 for cut-off values $10 \%, 8 \%$, and $6 \%$, respectively.

Table 16. Results of Counterfactual Analyses for MDJ (10\% Cut-off)

\begin{tabular}{|c|c|c|c|c|c|c|c|c|}
\hline \multirow{2}{*}{$\begin{array}{c}\text { Disease } \\
\text { name }\end{array}$} & \multicolumn{4}{|c|}{ MDJ-not-KAA } & \multicolumn{4}{|c|}{ MDJ-KAA } \\
\hline & $\mathrm{a}$ & $\mathrm{b}$ & $\mathrm{c}$ & $\mathrm{d}$ & $\mathrm{a}$ & $\mathrm{b}$ & $\mathrm{c}$ & $\mathrm{d}$ \\
\hline SEP & 936 & 722 & 105 & 62 & 521 & 1,137 & 75 & 92 \\
\hline $\mathrm{ARF}$ & 1,232 & 965 & 95 & 35 & 720 & 1,477 & 63 & 67 \\
\hline $\mathrm{CHF}$ & 1,239 & 1,314 & 65 & 39 & 807 & 1,746 & 43 & 61 \\
\hline PNE & 789 & 545 & 176 & 89 & 435 & 899 & 116 & 149 \\
\hline FED & 504 & 600 & 1,057 & 756 & 504 & 600 & 1,057 & 756 \\
\hline LD & 408 & 429 & 108 & 37 & 233 & 604 & 72 & 73 \\
\hline $\mathrm{HCSH}$ & 305 & 418 & 512 & 244 & 305 & 418 & 512 & 244 \\
\hline DA & 1,070 & 993 & 182 & 109 & 638 & 1,425 & 119 & 172 \\
\hline $\mathrm{MD}$ & 196 & 202 & 360 & 170 & 112 & 286 & 228 & 302 \\
\hline $\mathrm{EH}$ & 1,241 & 1,286 & 225 & 164 & 992 & 1,535 & 182 & 207 \\
\hline $\mathrm{CAH}$ & 803 & 926 & 440 & 215 & 549 & 1,180 & 258 & 397 \\
\hline DWC & 788 & 748 & 73 & 43 & 448 & 1,088 & 45 & 71 \\
\hline DLM & 533 & 674 & 586 & 317 & 533 & 674 & 586 & 317 \\
\hline DMC & 382 & 338 & 159 & 60 & 382 & 338 & 159 & 60 \\
\hline $\mathrm{CD}$ & 954 & 1,177 & 385 & 214 & 599 & 1,532 & 254 & 345 \\
\hline NSD & 558 & 437 & 341 & 135 & 325 & 670 & 233 & 243 \\
\hline HVD & 406 & 435 & 303 & 139 & 235 & 606 & 172 & 270 \\
\hline ED & 266 & 355 & 488 & 281 & 266 & 355 & 488 & 281 \\
\hline HD & 15 & 9 & 4 & 2 & 15 & 9 & 4 & 2 \\
\hline $\mathrm{RF}$ & 368 & 476 & 811 & 529 & 205 & 639 & 501 & 839 \\
\hline $\mathrm{MM}$ & 15 & 9 & 11 & 4 & 8 & 16 & 8 & 7 \\
\hline CKD & 326 & 440 & 564 & 229 & 190 & 576 & 380 & 413 \\
\hline SLE & 36 & 34 & 8 & 6 & 19 & 51 & 6 & 8 \\
\hline NECS & 18 & 13 & 58 & 20 & 18 & 13 & 58 & 20 \\
\hline CIBD & 28 & 44 & 29 & 10 & 23 & 49 & 26 & 13 \\
\hline MOS & 34 & 26 & 18 & 7 & 34 & 26 & 18 & 7 \\
\hline MS & 29 & 15 & 6 & 2 & 27 & 17 & 6 & 2 \\
\hline CNS & 19 & 15 & 8 & 8 & 19 & 15 & 8 & 8 \\
\hline LYM & 2 & 3 & 34 & 8 & 2 & 3 & 34 & 8 \\
\hline CRA & 11 & 12 & 14 & 3 & 11 & 12 & 14 & 3 \\
\hline Overall Diseases & 13,511 & 13,660 & 7,225 & 3,937 & 9,175 & 17,996 & 5,725 & 5,437 \\
\hline Rare Diseases & 207 & 180 & 190 & 70 & 176 & 211 & 182 & 78 \\
\hline
\end{tabular}


Table 17. Results of Counterfactual Analyses for MDJ (8\% Cut-off)

\begin{tabular}{|c|c|c|c|c|c|c|c|c|}
\hline \multirow{2}{*}{$\begin{array}{l}\text { Disease } \\
\text { name }\end{array}$} & \multicolumn{4}{|c|}{ MDJ-not-KAA } & \multicolumn{4}{|c|}{ MDJ-KAA } \\
\hline & $\mathrm{a}$ & $\mathrm{b}$ & $\mathrm{c}$ & $\mathrm{d}$ & $\mathrm{a}$ & $\mathrm{b}$ & $\mathrm{c}$ & $\mathrm{d}$ \\
\hline SEP & 936 & 722 & 105 & 62 & 468 & 1,190 & 63 & 104 \\
\hline $\mathrm{ARF}$ & 1,231 & 966 & 95 & 35 & 644 & 1,553 & 58 & 72 \\
\hline $\mathrm{CHF}$ & 1,239 & 1,314 & 65 & 39 & 735 & 1,818 & 38 & 66 \\
\hline PNE & 789 & 545 & 176 & 89 & 384 & 950 & 105 & 160 \\
\hline FED & 504 & 600 & 1,057 & 756 & 504 & 600 & 1,057 & 756 \\
\hline LD & 407 & 430 & 108 & 37 & 220 & 617 & 68 & 77 \\
\hline $\mathrm{HCSH}$ & 305 & 418 & 512 & 244 & 305 & 418 & 512 & 244 \\
\hline DA & 1,070 & 993 & 182 & 109 & 558 & 1,505 & 105 & 186 \\
\hline MD & 196 & 202 & 360 & 170 & 94 & 304 & 197 & 333 \\
\hline $\mathrm{EH}$ & 1,240 & 1,287 & 225 & 164 & 951 & 1,576 & 177 & 212 \\
\hline $\mathrm{CAH}$ & 803 & 926 & 440 & 215 & 522 & 1,207 & 245 & 410 \\
\hline DWC & 788 & 748 & 73 & 43 & 419 & 1,117 & 40 & 76 \\
\hline DLM & 533 & 674 & 586 & 317 & 533 & 674 & 586 & 317 \\
\hline DMC & 382 & 338 & 159 & 60 & 382 & 338 & 159 & 60 \\
\hline $\mathrm{CD}$ & 952 & 1,179 & 385 & 214 & 556 & 1,575 & 237 & 362 \\
\hline NSD & 558 & 437 & 341 & 135 & 288 & 707 & 210 & 266 \\
\hline HVD & 406 & 435 & 303 & 139 & 223 & 618 & 159 & 283 \\
\hline ED & 266 & 355 & 488 & 281 & 266 & 355 & 488 & 281 \\
\hline HD & 15 & 9 & 4 & 2 & 15 & 9 & 4 & 2 \\
\hline $\mathrm{RF}$ & 368 & 476 & 811 & 529 & 184 & 660 & 448 & 892 \\
\hline MM & 15 & 9 & 11 & 4 & 8 & 16 & 8 & 7 \\
\hline CKD & 326 & 440 & 564 & 229 & 175 & 591 & 346 & 447 \\
\hline SLE & 36 & 34 & 8 & 6 & 17 & 53 & 5 & 9 \\
\hline NECS & 18 & 13 & 58 & 20 & 18 & 13 & 58 & 20 \\
\hline CIBD & 28 & 44 & 29 & 10 & 22 & 50 & 25 & 14 \\
\hline MOS & 34 & 26 & 18 & 7 & 34 & 26 & 18 & 7 \\
\hline MS & 29 & 15 & 6 & 2 & 27 & 17 & 6 & 2 \\
\hline CNS & 19 & 15 & 8 & 8 & 19 & 15 & 8 & 8 \\
\hline LYM & 2 & 3 & 34 & 8 & 2 & 3 & 34 & 8 \\
\hline CRA & 11 & 12 & 14 & 3 & 11 & 12 & 14 & 3 \\
\hline Overall Diseases & 13,506 & 13,665 & 7,225 & 3,937 & 8,584 & 18,587 & 5,478 & 5,684 \\
\hline Rare Diseases & 207 & 180 & 190 & 70 & 173 & 214 & 180 & 80 \\
\hline
\end{tabular}


Table 18. Results of Counterfactual Analyses for MDJ (6\% Cut-off)

\begin{tabular}{|c|c|c|c|c|c|c|c|c|}
\hline \multirow{2}{*}{$\begin{array}{c}\text { Disease } \\
\text { name }\end{array}$} & \multicolumn{4}{|c|}{ MDJ-not-KAA } & \multicolumn{4}{|c|}{ MDJ-KAA } \\
\hline & $\mathrm{a}$ & $\mathrm{b}$ & $\mathrm{c}$ & $\mathrm{d}$ & $\mathrm{a}$ & $\mathrm{b}$ & $\mathrm{c}$ & $\mathrm{d}$ \\
\hline SEP & 935 & 723 & 105 & 62 & 425 & 1,233 & 57 & 110 \\
\hline ARF & 1,228 & 969 & 95 & 35 & 605 & 1,592 & 56 & 74 \\
\hline $\mathrm{CHF}$ & 1,238 & 1,315 & 65 & 39 & 691 & 1,862 & 36 & 68 \\
\hline PNE & 788 & 546 & 176 & 89 & 355 & 979 & 101 & 164 \\
\hline FED & 504 & 600 & 1,056 & 757 & 504 & 600 & 1,056 & 757 \\
\hline LD & 407 & 430 & 108 & 37 & 168 & 669 & 53 & 92 \\
\hline $\mathrm{HCSH}$ & 305 & 418 & 512 & 244 & 305 & 418 & 512 & 244 \\
\hline DA & 1,070 & 993 & 182 & 109 & 512 & 1,551 & 100 & 191 \\
\hline MD & 196 & 202 & 359 & 171 & 80 & 318 & 167 & 363 \\
\hline $\mathrm{EH}$ & 1,239 & 1,288 & 225 & 164 & 866 & 1,661 & 162 & 227 \\
\hline $\mathrm{CAH}$ & 802 & 927 & 440 & 215 & 481 & 1,248 & 232 & 423 \\
\hline DWC & 787 & 749 & 73 & 43 & 371 & 1,165 & 34 & 82 \\
\hline DLM & 531 & 676 & 586 & 317 & 531 & 676 & 586 & 317 \\
\hline DMC & 382 & 338 & 159 & 60 & 382 & 338 & 159 & 60 \\
\hline $\mathrm{CD}$ & 951 & 1,180 & 385 & 214 & 475 & 1,656 & 187 & 412 \\
\hline NSD & 558 & 437 & 341 & 135 & 225 & 770 & 174 & 302 \\
\hline HVD & 406 & 435 & 303 & 139 & 204 & 637 & 133 & 309 \\
\hline ED & 266 & 355 & 488 & 281 & 266 & 355 & 488 & 281 \\
\hline HD & 15 & 9 & 4 & 2 & 15 & 9 & 4 & 2 \\
\hline $\mathrm{RF}$ & 368 & 476 & 811 & 529 & 143 & 701 & 360 & 980 \\
\hline MM & 15 & 9 & 11 & 4 & 6 & 18 & 7 & 8 \\
\hline CKD & 326 & 440 & 564 & 229 & 157 & 609 & 309 & 484 \\
\hline SLE & 36 & 34 & 8 & 6 & 15 & 55 & 5 & 9 \\
\hline NECS & 18 & 13 & 58 & 20 & 18 & 13 & 58 & 20 \\
\hline CIBD & 28 & 44 & 29 & 10 & 20 & 52 & 25 & 14 \\
\hline MOS & 34 & 26 & 18 & 7 & 34 & 26 & 18 & 7 \\
\hline MS & 29 & 15 & 6 & 2 & 24 & 20 & 5 & 3 \\
\hline CNS & 19 & 15 & 8 & 8 & 19 & 15 & 8 & 8 \\
\hline LYM & 2 & 3 & 34 & 8 & 2 & 3 & 34 & 8 \\
\hline CRA & 11 & 12 & 14 & 3 & 11 & 12 & 14 & 3 \\
\hline Overall Diseases & 13,494 & 13,677 & 7,223 & 3,939 & 7,910 & 19,261 & 5,140 & 6,022 \\
\hline Rare Diseases & 207 & 180 & 190 & 70 & 164 & 223 & 178 & 82 \\
\hline
\end{tabular}




\section{REFERENCES}

[1] Alan R. Aronson. 2001. Effective mapping of biomedical text to the UMLS metathesaurus: The metamap program. In Proceedings of the AMIA Symposium. American Medical Informatics Association, 17.

[2] Eta S. Berner. 2007. Clinical Decision Support Systems. Vol. 233. Springer.

[3] Sakyajit Bhattacharya, Vijay Huddar, Vaibhav Rajan, and Chandan K. Reddy. 2018. A dual boundary classifier for predicting acute hypotensive episodes in critical care. PloS One 13, 2 (2018).

[4] Antoine Bordes, Nicolas Usunier, Alberto Garcia-Duran, Jason Weston, and Oksana Yakhnenko. 2013. Translating embeddings for modeling multi-relational data. In Proceedings of the International Conference on Advances in Neural Information Processing Systems. 2787-2795.

[5] Matthew R. Boutell, Jiebo Luo, Xipeng Shen, and Christopher M. Brown. 2004. Learning multi-label scene classification. Pattern Recog. 37, 9 (2004), 1757-1771.

[6] John A. Bridgewater, Karyn A. Goodman, Aparna Kalyan, and Mary F. Mulcahy. 2016. Biliary tract cancer: Epidemiology, radiotherapy, and molecular profiling. Amer. Soc. Clin. Oncol. Educ. Book 36 (2016), e194-e203.

[7] U.S. Census Bureau. 2015. Annual Estimates of the Resident Population for Selected Age Groups by Sex for the United States, States, Counties and Puerto Rico Commonwealth and Municipios: April 1, 2010 to July 1, 2015. Retrieved from https://www.census.gov/data/tables/time-series/demo/popest/2010s-national-detail.html.

[8] CDC. 2014. National Diabetes Statistics Report, Center for Disease Control and Prevention. Retrieved from http: //www.cdc.gov/diabetes/pdfs/data/2014-report-estimates-of-diabetes-and-its-burden-in-the-united-states.pdf.

[9] CDN. 2014. Rare Disease Facts, National Organization for Rare Disorders. Retrieved from https://www.nidcd.nih. gov/directory/national-organization-rare-disorders-nord\#: :text=Description\%3A, affects\%20fewer\%20than\%20200 $\% 2 \mathrm{C} 000 \% 20$ Americans.

[10] Zhengping Che, David Kale, Wenzhe Li, Mohammad Taha Bahadori, and Yan Liu. 2015. Deep computational phenotyping. In Proceedings of the 21st ACM SIGKDD International Conference on Knowledge Discovery and Data Mining. 507-516.

[11] Edward Choi, Mohammad Taha Bahadori, Andy Schuetz, Walter F. Stewart, and Jimeng Sun. 2016. Doctor AI: Predicting clinical events via recurrent neural networks. In Proceedings of the Machine Learning for Healthcare Conference. 301-318.

[12] Edward Choi, Mohammad Taha Bahadori, Le Song, Walter F. Stewart, and Jimeng Sun. 2017. GRAM: Graph-based attention model for healthcare representation learning. In Proceedings of the 23rd ACM SIGKDD International Conference on Knowledge Discovery and Data Mining. 787-795.

[13] Edward Choi, Mohammad Taha Bahadori, Jimeng Sun, Joshua Kulas, Andy Schuetz, and Walter Stewart. 2016. Retain: An interpretable predictive model for healthcare using reverse time attention mechanism. In Proceedings of the International Conference on Advances in Neural Information Processing Systems. 3504-3512.

[14] Edward Choi, Cao Xiao, Walter Stewart, and Jimeng Sun. 2018. MiME: Multilevel medical embedding of electronic health records for predictive healthcare. In Proceedings of the International Conference on Advances in Neural Information Processing Systems. 4547-4557.

[15] N. Danchenko, J. A. Satia, and M. S. Anthony. 2006. Epidemiology of systemic lupus erythematosus: A comparison of worldwide disease burden. Lupus 15, 5 (2006), 308-318.

[16] Mandip S. Dhamoon and Mitchell S. V. Elkind. 2010. Inclusion of stroke as an outcome and risk equivalent in risk scores for primary and secondary prevention of vascular disease. Circulation 121, 18 (2010), 2071-2078.

[17] Yuan Fang, Kingsley Kuan, Jie Lin, Cheston Tan, and Vijay Chandrasekhar. 2017. Object detection meets knowledge graphs. In Proceedings of the 26th International foint Conference on Artificial Intelligence (IFCAI'17).

[18] Jessie Gerteis, David Izrael, Deborah Deitz, Lisa LeRoy, Richard Ricciardi, Therese Miller, and Jayasree Basu. 2014. Multiple Chronic Conditions Chartbook. Agency for Healthcare Research and Quality, Rockville, MD.

[19] Deborah Grady and Seth A. Berkowitz. 2011. Why is a good clinical prediction rule so hard to find? Arch. Internal Medic. 171, 19 (2011), 1701-1702.

[20] Rebecca S. Graves. 2002. Users' guides to the medical literature: A manual for evidence-based clinical practice. $\mathcal{F}$. Medic. Libr. Assoc. 90, 4 (2002), 483.

[21] Shirley Gregor and Alan R. Hevner. 2013. Positioning and presenting design science research for maximum impact. MIS Quart. 37, 2 (2013), 337-355.

[22] Trevor Hastie, Robert Tibshirani, and Jerome Friedman. 2009. The Elements of Statistical Learning: Data Mining, Inference, and Prediction. Springer Science \& Business Media.

[23] HCUP. 2020. Clinical Classications Software (CCS) for ICD-9-CM Fact Sheet, Agency for Healthcare Research and Quality. Retrieved from https://www.hcup-us.ahrq.gov/ toolssoftware/ccs/ccsfactsheet.jsp.

[24] Vijay Huddar, Bapu Koundinya Desiraju, Vaibhav Rajan, Sakyajit Bhattacharya, Shourya Roy, and Chandan K. Reddy. 2016. Predicting complications in critical care using heterogeneous clinical data. IEEE Access 4 (2016), 7988-8001.

[25] IDF. 2015. What Is Diabetes? Retrieved from https://www.idf.org/about-diabetes/what-is-diabetes.html. 
[26] Shaoxiong Ji, Shirui Pan, Erik Cambria, Pekka Marttinen, and Philip S. Yu. 2020. A survey on knowledge graphs: Representation, acquisition and applications. arXiv preprint arXiv:2002.00388 (2020).

[27] Alistair E. W. Johnson, Tom J. Pollard, Lu Shen, H. Lehman Li-wei, Mengling Feng, Mohammad Ghassemi, Benjamin Moody, Peter Szolovits, Leo Anthony Celi, and Roger G. Mark. 2016. MIMIC-III, a freely accessible critical care database. Sci. Data 3 (2016), 160035.

[28] Halil Kilicoglu, Dongwook Shin, Marcelo Fiszman, Graciela Rosemblat, and Thomas C. Rindflesch. 2012. SemMedDB: A PubMed-scale repository of biomedical semantic predications. Bioinformatics 28, 23 (2012), 3158-3160.

[29] M. S. V. Elkind, R. D’Agostino, M. S. Dhamoon, D. T. Lackland, D. C. Goff, R. T. Higashida, L. A. McClure, P. H. Mitchell, R. L. Sacco, C. A. Sila, S. C. Smith, D. Tanne, D. L. Tirschwell, E. Touzé, and L. R. Wechsler. 2012. Inclusion of stroke in cardiovascular risk prediction instruments a statement for healthcare professionals from the American Heart Association/American Stroke Association. Stroke 43, 7 (2012), 1998-2027.

[30] Yu-Kai Lin, Hsinchun Chen, Randall A. Brown, Shu-Hsing Li, and Hung-Jen Yang. 2017. Healthcare predictive analytics for risk profiling in chronic care: A Bayesian multitask learning approach. MIS Quart. 41, 2 (2017).

[31] Zachary C. Lipton, David C. Kale, Charles Elkan, and Randall Wetzel. 2016. Learning to diagnose with LSTM recurrent neural networks. In Proceedings of the International Conference on Learning Representations.

[32] Xu-Ying Liu, Jianxin Wu, and Zhi-Hua Zhou. 2008. Exploratory undersampling for class-imbalance learning. IEEE Trans. Syst. Man Cyber. Part B (Cyber.) 39, 2 (2008), 539-550.

[33] Fenglong Ma, Radha Chitta, Jing Zhou, Quanzeng You, Tong Sun, and Jing Gao. 2017. Dipole: Diagnosis prediction in healthcare via attention-based bidirectional recurrent neural networks. In Proceedings of the 23rd ACM SIGKDD International Conference on Knowledge Discovery and Data Mining. 1903-1911.

[34] Fenglong Ma, Quanzeng You, Houping Xiao, Radha Chitta, Jing Zhou, and Jing Gao. 2018. KAME: Knowledge-based attention model for diagnosis prediction in healthcare. In Proceedings of the 27th ACM International Conference on Information and Knowledge Management. 743-752.

[35] Justin Mower, Devika Subramanian, and Trevor Cohen. 2018. Learning predictive models of drug side-effect relationships from distributed representations of literature-derived semantic predications. f. Amer. Medic. Inform. Assoc. 25, 10, 1339-1350.

[36] Mark A. Musen, Blackford Middleton, and Robert A. Greenes. 2014. Clinical decision-support systems. In Biomedical Informatics. Springer, 643-674.

[37] United Nations. 2015. The World Population Prospects: 2015 Revision. Retrieved from https://www.un.org/en/ development/desa/publications/world-population-prospects-2015-revision.html.

[38] NLM. 2019. MEDLINE, U.S. National Library of Medicine. Retrieved from https://www.nlm.nih.gov/bsd/medline.html.

[39] Narges Razavian, Jake Marcus, and David Sontag. 2016. Multi-task prediction of disease onsets from longitudinal laboratory tests. In Proceedings of the Machine Learning for Healthcare Conference. 73-100.

[40] Jesse Read, Bernhard Pfahringer, Geoff Holmes, and Eibe Frank. 2011. Classifier chains for multi-label classification. Mach. Learn. 85, 3 (2011), 333.

[41] K. Srinath Reddy. 2016. Global burden of disease study 2015 provides GPS for global health 2030. Lancet 388, 10053 (2016), 1448-1449.

[42] Junyuan Shang, Shenda Hong, Yuxi Zhou, Meng Wu, and Hongyan Li. 2018. Knowledge guided multi-instance multilabel learning via neural networks in medicines prediction. In Proceedings of the Asian Conference on Machine Learning. 831-846.

[43] Benjamin Shickel, Patrick James Tighe, Azra Bihorac, and Parisa Rashidi. 2017. Deep EHR: A survey of recent advances in deep learning techniques for electronic health record (EHR) analysis. IEEE J. Biomed. Health Inform. 22, 5 (2017), 1589-1604.

[44] Vergil N. Slee. 1978. The International Classification of Diseases: Ninth Revision (ICD-9). Retrieved from https://www. cdc.gov/nchs/icd/icd9cm.htm.

[45] Michael Steinbach, George Karypis, Vipin Kumar et al. 2000. A Comparison of Document Clustering Techniques KDD Workshop on Text Mining. Retrieved from https://hdl.handle.net/11299/215421.

[46] Shire Human Genetic Therapies. 2013. Rare Disease Impact Report: Insights from Patients and the Medical Community. Technical Report. Shire Human Genetic Therapies.

[47] Philip J. Vickers. 2013. Challenges and opportunities in the treatment of rare diseases. Drug Discov. World 14 (2013), $9-16$.

[48] Quan Wang, Zhendong Mao, Bin Wang, and Li Guo. 2017. Knowledge graph embedding: A survey of approaches and applications. IEEE Trans. Knowl. Data Eng. 29, 12 (2017), 2724-2743.

[49] Agnieszka Wosiak, Kinga Glinka, and Danuta Zakrzewska. 2018. Multi-label classification methods for improving comorbidities identification. Comput. Biol. Med. 100 (2018), 279-288.

[50] Xi-Zhu Wu and Zhi-Hua Zhou. 2017. A unified view of multi-label performance measures. In Proceedings of the 34th International Conference on Machine Learning, Vol. 70. JMLR.org, 3780-3788. 
[51] Pranjul Yadav, Michael Steinbach, Vipin Kumar, and Gyorgy Simon. 2018. Mining electronic health records (EHRs) a survey. ACM Comput. Surv. 50, 6 (2018), 1-40.

[52] Changchang Yin, Rongjian Zhao, Buyue Qian, Xin Lv, and Ping Zhang. 2019. Domain knowledge guided deep learning with electronic health records. In Proceedings of the IEEE International Conference on Data Mining (ICDM'19). IEEE, 738-747.

[53] Min-Ling Zhang and Zhi-Hua Zhou. 2007. ML-KNN: A lazy learning approach to multi-label learning. Pattern Recog. 40, 7 (2007), 2038-2048.

[54] Min-Ling Zhang and Zhi-Hua Zhou. 2013. A review on multi-label learning algorithms. IEEE Trans. Knowl. Data Eng. 26, 8 (2013), 1819-1837.

[55] Jiayu Zhou, Jimeng Sun, Yashu Liu, Jianying Hu, and Jieping Ye. 2013. Patient risk prediction model via top-k stability selection. In Proceedings of the SIAM International Conference on Data Mining. SIAM, 55-63.

[56] Donald W. Zimmerman. 1997. Teacher's corner: A note on interpretation of the paired-samples T test. F. Educ. Behav. Statist. 22, 3 (1997), 349-360.

[57] Damien Zufferey, Thomas Hofer, Jean Hennebert, Michael Schumacher, Rolf Ingold, and Stefano Bromuri. 2015. Performance comparison of multi-label learning algorithms on clinical data for chronic diseases. Comput. Biol. Med. 65 (2015), 34-43.

Received June 2020; revised January 2021; accepted January 2021 